TRANSACTIONS OF THE

AMERICAN MATHEMATICAL SOCIETY

Volume 356, Number 5, Pages 2093-2121

S 0002-9947(04)03526-3

Article electronically published on January 6, 2004

\title{
CONVERGENCE OF SINGULAR LIMITS FOR MULTI-D SEMILINEAR HYPERBOLIC SYSTEMS TO PARABOLIC SYSTEMS
}

\author{
DONATELLA DONATELLI AND PIERANGELO MARCATI
}

\begin{abstract}
In this paper we investigate the diffusive zero-relaxation limit of the following multi-D semilinear hyperbolic system in pseudodifferential form: $W_{t}(x, t)+\frac{1}{\varepsilon} A(x, D) W(x, t)=\frac{1}{\varepsilon^{2}} B(x, W(x, t))+\frac{1}{\varepsilon} D(W(x, t))+E(W(x, t))$. We analyze the singular convergence, as $\varepsilon \downarrow 0$, in the case which leads to a limit system of parabolic type. The analysis is carried out by using the following steps:

(i) We single out algebraic "structure conditions" on the full system, motivated by formal asymptotics, by some examples of discrete velocity models in kinetic theories.

(ii) We deduce "energy estimates", uniformly in $\varepsilon$, by assuming the existence of a symmetrizer having the so-called block structure and by assuming "dissipativity conditions" on $B$.

(iii) We assume a Kawashima type condition and perform the convergence analysis by using generalizations of compensated compactness due to Tartar and Gérard.

Finally, we include examples that show how to use our theory to approximate any quasilinear parabolic systems, satisfying the Petrowski parabolicity condition, or general reaction diffusion systems, including Chemotaxis and Brusselator type systems.
\end{abstract}

\section{INTRODUCTION}

In this paper we study the semilinear multidimensional hyperbolic system with a small parameter $\varepsilon>0$,

$$
W_{t}(x, t)+\frac{1}{\varepsilon} A(x, D) W(x, t)=\frac{1}{\varepsilon^{2}} B(x, W(x, t))+\frac{1}{\varepsilon} D(W(x, t))+E(W(x, t)),
$$

where $W=W(x, t) \in \mathbb{R}^{N}, x \in \mathbb{R}^{d}, t \geq 0$, and $A(x, D)$ is a first-order pseudodifferential operator. The system (1.1) includes the case of first-order differential

Received by the editors July 15, 2002 and, in revised form, March 26, 2003 and June 18, 2003. 2000 Mathematics Subject Classification. Primary 35L40, 35K40; Secondary 58J45, 58J37.

Key words and phrases. Hyperbolic systems, parabolic systems, pseudodifferential operators, relaxation theory.

This research was partially supported by EU financed network no. HPRN-CT-2002-00282 and by COFIN MIUR 2002 "Equazioni paraboliche e iperboliche nonlineari". 
operators of the form

$$
W_{t}(x, t)+\frac{1}{\varepsilon} \sum_{j=1}^{d} A_{j}(x) \partial_{j} W(x, t)=\frac{1}{\varepsilon^{2}} B(x, W(x, t))+\frac{1}{\varepsilon} D(W(x, t))+E(W(x, t)),
$$

where for any $x \in \mathbb{R}^{d}, A_{j}(x), j=1, \ldots, d$, are $N \times N$ matrices. Our aim is to describe the limiting behaviour of the system (1.1) as $\varepsilon$ goes to zero. We look for structure conditions to ensure that (1.1) approximates in the limit a second-order parabolic system. Our interest in this problem is motivated also by a very strong similarity with the limiting structure appearing in the investigation of the hydrodynamic limit for the Boltzmann equation, in particular, in the discrete velocity case. The Boltzmann equation describes the evolution of the density $f(x, \xi, t)$ of particles which is a function of time $t$, space $x$ and velocity $\xi$ and has the form

$$
\nu f_{t}+\xi \nabla \cdot f=\frac{1}{\varepsilon} Q(f, f),
$$

where $\nu$ is the Mach number and $\varepsilon$ the Knudsen number. By averaging $f(x, \xi, t)$ in $\xi$ and by using higher order momenta we can define a hierarchy of macroscopic quantities. The investigation of the hydrodynamic limit regards the behaviour of those quantities (actually combined with the closure problems as the Knudsen number goes to zero). In the case where the Mach number is of the same order as the Knudsen number our limit can be described by the Navier Stokes equation; otherwise, when the Mach number is fixed and the Knudsen number tends to zero we end up with the Euler equation. Those limits allow us to understand the differences between relaxation limits of hyperbolic type to parabolic with respect to those of hyperbolic to hyperbolic type [3], [6], [14, [19], 20], 21], 34], [58. In particular, if we deal with discrete velocity models, the equivalent "Boltzmann equation" is a semilinear hyperbolic system. The simplest example is given by Carleman's equation

$$
\left\{\begin{array}{l}
f_{1 t}+\frac{1}{\varepsilon} f_{1 x}=\frac{1}{\varepsilon^{2}}\left(f_{2}^{2}-f_{1}^{2}\right), \\
f_{2 t}-\frac{1}{\varepsilon} f_{2 x}=\frac{1}{\varepsilon^{2}}\left(f_{1}^{2}-f_{2}^{2}\right),
\end{array}\right.
$$

where we take $\xi \in\{-1,1\}$ and $f_{1}=f(x, 1, t), f_{2}=f(x,-1, t)$. By rescaling the variable we get that $\rho=f_{1}+f_{2}$ as $\varepsilon \downarrow 0$ satisfies the nonlinear diffusion equation

$$
\rho_{t}=\frac{1}{2}(\log \rho)_{x x} .
$$

This asymptotic problem was first investigated by Kurtz [26] and McKean [2]. Therefore, the nonlinear diffusion problem, obtained as the limit of the Cattaneo hyperbolic nonlinear heat conduction equation, was proved by Marcati, Milani and Secchi [38]. The paper of Marcati and Milani [37] concerns the porous media flow as the limit of the Euler equation in 1-D, later generalized by Marcati and Rubino [41] to the multi-D case. Relaxation phenomena of the same nature appear in the zero relaxation limits for the Euler-Poisson model for the semiconductor devices and it was investigated by Marcati and Natalini [39], [40] in the 1-D case and by Lattanzio and Marcati [27] in the multi-D case. More recently Lions and Toscani [33] investigated a discrete velocity model leading to the porous media flow by making the same use of compensated compactness as done in [37]. All 
of these papers, with the exception of [26], [42], make use of the techniques of compensated compactness. Similar ideas have been applied by Marcati and Rubino [41] to show the general theory for $2 \times 2$ systems in the 1 -D case and to propose a general framework that we are going to investigate here in the semilinear system case. Models of BGK type approximation have been successfully studied in this framework by Bouchut, Guarguaglini and Natalini [1] and Lattanzio, Natalini [28] in the case of 1-D systems. The framework of 41 was also investigated in the quasilinear case by Lattanzio and Yong [29] for $H^{s}$-smooth solutions. Recently, with a similar approach, Junk and Yong [18] derived the incompressible Navier-Stokes equations from the BGK model. Preliminary results concerning semilinear systems have been obtained in [9], [10; in particular, in [10] we considered a 1-D semilinear system with variable coefficients. Already in these cases the classical compensated compactness is not sufficient and it is necessary to use a generalized version of this theory due to Tartar 54] and P. Gérard [13. From the technical point of view, the theory has been developed in the pseudodifferential setting since in any case (differential and pseudodifferential ones), we are obliged to use pseudodifferential symmetrizers.

The plan of the paper is as follows. Section 2 provides the basic definitions and notation, and, moreover, recalls some mathematical tools needed later. In Section 3 we describe the structural conditions on our system and on its symmetrizers and we describe the formal framework of the limiting process. The parabolicity conditions for the limit system are a direct consequence of the previous assumptions. Section 4 is devoted to showing a rigorous proof of the previous formal analysis. In particular, the assumptions of the previous sections allow us to prove "a priori" estimates of energy type, uniformly in $\varepsilon$. By only using the information provided by the energy estimates (and no $L^{\infty}$ bound) we will be able to obtain our relaxation results by means of the previously mentioned compactness framework. In Section 5 we show how our theory can be naturally simplified in the case of constant coefficients. Finally, in Section 6 we show how to use the theory developed in the previous sections in order to approximate any prescribed nonlinear parabolic system, by means of a (larger) hyperbolic system with semilinear structure. We will approximate nonlinear parabolic systems in divergence form of the type

$$
U_{t}+\sum_{i=1}^{d} \partial_{i}\left(F_{i}(U)-\sum_{j=1}^{d} B_{i j}(U) \partial_{j} U\right)=G(U)
$$

where $x \in \mathbb{R}^{d}, t \in \mathbb{R}_{+}, U=U(x, t) \in \mathbb{R}^{k}$. With the previous techniques we also approximate reaction-diffusion type systems of the form

$$
U_{t}=\sum_{j, k=1}^{d} A_{j, k}(x) \partial_{j} \partial_{k} U+f(U),
$$

where $x \in \mathbb{R}^{d}, t \in \mathbb{R}_{+}, U=U(x, t) \in \mathbb{R}^{k}$. In this latter case we use two distinct approaches. The first is based on the theory of pseudodifferential operators (roughly speaking, a square root type argument) while the second is based on symmetric differential operators and can be more useful for numerical computations. Additional examples are given by the Chemotaxis and the Brusselator systems. We remark that this latter approximation extends in the multi-D framework those proposed by Jin and Liu [16] and Lattanzio and Natalini [28] (example 5.3). Our theory does 
not require $L^{\infty}$ estimates (for instance, via invariant domains as in Serre [51]). Of course this fact restricts our attention only to Lipschitz functions in the right-hand side of (1.1), so that we always have global existence. For numerical application we refer, for instance, to [17] or [46].

\section{Preliminary NOTIONS}

We start this section by introducing the main notation and definitions used in this article. In particular, we recall some basic facts and notation concerning the theory of pseudodifferential operators and we also recall our main compactness tools used in the strong convergence analysis. Namely

(a) $(\cdot, \cdot)$ denotes the scalar product in $\mathbb{R}^{q}(q=1,2, \ldots)$ and $|\cdot|$ the usual norm of $\mathbb{R}^{q}(q=1,2, \ldots)$,

(b) $\mathcal{M}_{m \times n}$ denotes the linear space of $m \times n$ matrices,

(c) $(\cdot, \cdot)_{2}$ denotes the scalar product in $L^{2}\left(\mathbb{R}^{d}\right)$ and $\|\cdot\|$ the norm in $L^{2}\left(\mathbb{R}^{d}\right)$,

(d) $\mathcal{D}\left(\mathbb{R}^{d} \times \mathbb{R}_{+}\right)$denotes the space of test functions $C_{0}^{\infty}\left(\mathbb{R}^{d} \times \mathbb{R}_{+}\right), \mathcal{D}^{\prime}\left(\mathbb{R}^{d} \times\right.$ $\mathbb{R}_{+}$) the Schwartz space of distributions and $\langle\cdot, \cdot\rangle$ the duality bracket in $\mathcal{D}^{\prime}\left(\mathbb{R}^{d} \times \mathbb{R}_{+}\right)$,

(e) $H$ is a separable Hilbert space, $\mathcal{L}(H)$ the space of bounded operators, $\mathcal{K}(H)$ the space of compact operators,

(f) if $X, Y$ are Banach spaces, $\mathcal{L}(X, Y)$ is the space of bounded operators,

(g) we denote by $H_{l o c}^{s}(\Omega, H)$ the classical local Sobolev space of order $s$, i.e., $u \in H_{l o c}^{s}(\Omega, H) \Longleftrightarrow \forall \varphi \in C_{0}^{\infty},(\widehat{\varphi u}) \in L^{2}\left(\mathbb{R}^{n},\left(1+|\xi|^{2}\right)^{s} d \xi\right)$,

(h) we denote by $F_{I I}\left(x, Z^{I}, Z^{I I}\right)$ the derivative with respect to the variable $Z^{I I}$ of $F\left(x, Z^{I}, Z^{I I}\right)$.

We shall also use the notion of parabolicity for systems of equations in various ways (see Taylor [57, volume III, [56], Eidel'man [11], Kreiss and Lorenz [25]). Let us consider the system

$$
u_{t}=\sum_{j, k} A^{j, k}\left(t, x, D_{x}^{\prime} u\right) \partial_{j} \partial_{k} u+B\left(t, x, D_{x}^{\prime} u\right),
$$

where $u \in \mathbb{R}^{p}, A^{j, k}\left(t, x, D_{x}^{\prime} u\right) \in \mathcal{M}_{p \times p}, B\left(t, x, D_{x}^{\prime} u\right) \in \mathbb{R}^{p}$ and $D_{x}^{\prime}$ is a differential operator of order not greater than two. The system is said to be strongly parabolic if there exists $c_{0}>0$, such that for all $\xi \in \mathbb{R}^{d}$ one has

$$
\sum_{j, k} A^{j, k}\left(t, x, D_{x}^{\prime} u\right) \xi_{j} \xi_{k} \geq c_{0}|\xi|^{2} I .
$$

Namely, if we denote $L\left(t, x, D_{x}^{\prime} u, \xi\right)=-\sum_{j, k} A^{j, k}\left(t, x, D_{x}^{\prime} u\right) \xi_{j} \xi_{k}$ this condition is equivalent to saying $L+L^{T}$ is a negative definite matrix. Unfortunately, this condition is very restrictive and it is not invariant under a frame change; therefore, we will use a more general notion often referred to as Petrowski parabolicity (see Taylor [57], volume III, [56]).

We say that the system (2.1) is parabolic in the sense of Petrowski if, with the eigenvalues of the matrix $L\left(t, x, D_{x}^{\prime} u, \xi\right)$ denoted by $\lambda_{k}\left(t, x, D_{x}^{\prime} u, \xi\right)$, there exists $\alpha_{0}>0$ such that, for all $\xi \in \mathbb{R}^{d}$,

$$
\operatorname{Re} \lambda_{k}\left(t, x, D_{x}^{\prime} u, \xi\right) \leq-\alpha_{0}|\xi|^{2} .
$$

The latter notion of parabolicity is equivalent to the existence of a symmetric matrix $P_{0}\left(t, x, D_{x}^{\prime} u, \xi\right)$, positive definite (i.e. $P_{0} \geq c I>0$ ), homogeneous of degree 0 in $\xi$, 
such that

$$
-\left(P_{0} L+L^{*} P_{0}\right) \geq c|\xi|^{2} I>0 .
$$

Let us recall the basic notation concerning pseudodifferential operators and symbols to be used later on. We refer to [56] for details. Assuming $\rho, \delta \in[0,1], m \in \mathbb{R}$, we denote by $S_{\rho, \delta}^{m}$ the set of $C^{\infty}$ symbols satisfying

$$
\left|D_{x}^{\beta} D_{\xi}^{\alpha} p(x, \xi)\right| \leq C_{\alpha, \beta}\langle\xi\rangle^{m-\rho|\alpha|+\delta|\beta|}
$$

for all $\alpha, \beta$, where $\langle\xi\rangle=\left(1+|\xi|^{2}\right)^{1 / 2}$. In such a case we say that the associated operator denoted by $O P(p(x, \xi))$ is given by

$$
P(x, D) f(x)=\int p(x, \xi) \hat{f}(\xi) e^{i x \xi} d \xi:=O P(p(x, \xi))
$$

(where $\hat{f}(\xi)=(2 \pi)^{-n} \int f(x) e^{-i x \xi} d x$ denotes the Fourier transform of the function $f)$ and belongs to $O P S_{\rho, \delta}^{m}$. If there are smooth symbols $p_{m-j}(x, \xi)$, homogeneous in $\xi$ of degree $m-j$ for $|\xi| \geq 1$, i.e., $p_{m-j}(x, r \xi)=r^{m-j} p_{m-j}(x, \xi)$ for $r,|\xi| \geq 1$, and if

$$
p(x, \xi) \sim \sum_{j \geq 0} p_{m-j}(x, \xi)
$$

in the sense that

$$
p(x, \xi)-\sum_{j \geq 0}^{N} p_{m-j}(x, \xi) \in S_{1,0}^{m-N}
$$

for all $N$, then we say $p(x, \xi) \in S^{m}$. We also define

$$
S^{-\infty}=\bigcap_{m>0} S_{\rho, \delta}^{-m}
$$

The following properties will also be used here.

Theorem 2.1 ([56], Theorem $0.5 \mathrm{~A})$. If $P(x, D) \in O P S_{\rho, \delta}^{0}$ and $0 \leq \delta<\rho \leq 1$, then

$$
P(x, D): L^{2}\left(\mathbb{R}^{n}\right) \longrightarrow L^{2}\left(\mathbb{R}^{n}\right)
$$

Theorem 2.2 ([55], Chapter II, Lemma 6.2 Ex. 8.1). If $P(x, D) \in O P S_{\rho, \delta}^{0}, \delta<\rho$, and if $\Re e p(x, \xi) \geq k>0$, then there exists $\widetilde{R}(x, D) \in O P S_{\rho, \delta}^{0}$ such that $\widetilde{R}(x, D) \geq$ $\eta I>0$, with

$$
\Re e P(x, D)=\frac{1}{2}\left(P+P^{*}\right), \quad \Re e P(x, D)-\widetilde{R}(x, D) \in O P S^{-\infty} .
$$

Proposition 2.3 (Commutator estimate) ([56], Proposition 3.6 B). Given $P(x, D) \in O P S_{1,0}^{0}$ we have

$$
\|[P, f] u\|_{W^{\sigma, p}} \leq k\|f\|_{L i p}\|u\|_{W^{\sigma-1, p}} \quad \text { for } 0 \leq \sigma \leq 1,
$$

in particular, if $\sigma=0$ and $p=2$, then

$$
\|[P, f] u\|_{L^{2}\left(\mathbb{R}^{n}\right)} \leq k\|f\|_{L i p}\|u\|_{H^{-1}} .
$$

Finally, we state here the compactness tool, due to Tartar and Gérard ([54], [13]). The classical results concerning the applications of compensated compactness in the theory of hyperbolic systems are reported in the books by Dafermos [5] and Serre [50]. Let $H, H^{\sharp}$ denote separable Hilbert spaces, let $\Omega \in \mathbb{R}^{n}$ be an open set, and let $m \in \mathbb{N}$. We have the following theorem taken from Gérard [13]. 
Theorem 2.4 (Compensated Compactness Principle). Let $P \in O P S^{m}$ with principal symbol $p(x, \xi)$, and let $\left\{u_{k}\right\}$ be a bounded sequence of $L_{\text {loc }}^{2}(\Omega, H)$ such that $u_{k} \rightarrow u$. Assume that there exists a dense subset $D \in H^{\sharp}$ such that, for any $h \in D$, the sequence $\left(\left\langle P u_{k}, h\right\rangle\right)$ is relatively compact in $H_{\text {loc }}^{-m}(\Omega)$. Moreover, let $\chi \in C(\Omega, \mathcal{K}(H))$.

(i) If $\chi=\chi^{*}$ and for all $(x, \eta, h) \in \Omega \times S^{n-1} \times H$, one has

$$
(p(x, \eta) h=0) \Rightarrow(\langle\chi(x) h, h\rangle \geq 0),
$$

then, for any nonnegative $\varphi \in C_{0}^{\infty}(\Omega)$,

$$
\liminf _{k \rightarrow \infty} \int_{\Omega} \varphi\left\langle\chi(x) u_{k}, u_{k}\right\rangle d x \geq \int_{\Omega} \varphi\langle\chi(x) u, u\rangle d x .
$$

(ii) If for $(x, \eta, h) \in \Omega \times S^{n-1} \times H$, one has

$$
(p(x, \eta) h=0) \Rightarrow(\langle\chi(x) h, h\rangle=0),
$$

then

$$
\left\langle\chi(x) u_{k}, u_{k}\right\rangle \quad \text { converges to } \quad\langle\chi(x) u, u\rangle \quad \text { in } \mathcal{D}^{\prime}(\Omega) \text {. }
$$

The previous theorem holds also if we replace a pseudodifferential operator $P \in$ $O P S^{m}$ with the differential operator in divergence form

$$
P u(x)=\sum_{|\alpha| \leq m} \partial^{\alpha}\left(a_{\alpha}(x) u(x)\right),
$$

where $\alpha \in \mathbb{N}^{n},|\alpha| \leq m, a_{\alpha} \in C\left(\Omega, \mathcal{L}\left(H, H^{\sharp}\right)\right)$.

\section{Structural FrameWORK}

In this section we shall explain how to decouple the system (1.1), the natural structural conditions needed for a nontrivial limiting process, the conditions imposed on the symmetrizers and the dissipative conditions on the nonlinear term $Q$. In conclusion we provide a formal limit analysis. To simplify the computations we shall restrict our analysis to the case $E(W) \equiv 0$, but our results can be easily extended to the case of nonzero $E(\cdot) \in \operatorname{Lip}\left(\mathbb{R}^{N}, \mathbb{R}^{N}\right)$.

3.1. Decoupled system. We will consider the semilinear system of equations

$$
W_{t}(x, t)+A(x, D) W(x, t)=B(x, W(x, t))+D(W(x, t)),
$$

where $t \geq 0, x \in \mathbb{R}^{d}, W \in \mathbb{R}^{N}$. We assume the following hypotheses hold.

(A.1) $B(\cdot, \cdot) \in C^{1}\left(\mathbb{R}^{d+N}, \mathbb{R}^{N}\right), D(\cdot) \in C^{1}\left(\mathbb{R}^{N}, \mathbb{R}^{N}\right)$.

(A.2) $A(x, D) \in O P S^{1}$, the system (3.1) is hyperbolic, namely the principal symbol of $A(x, D)$ is the matrix $a(x, \xi) \in \mathcal{M}_{N \times N}$ whose eigenvalues for $(x, \xi) \in \mathbb{R}^{d} \times \mathbb{R}^{d}, \xi \neq 0$, are purely imaginary.

(A.3) Let $S=\operatorname{span}\left\{B(x, W) \mid(x, W) \in \mathbb{R}^{d+N}\right\}$; then $\operatorname{dim} S=N-k, 0<k<$ $N$.

Remark 3.1. We point out that (3.1) includes the case of the hyperbolic semilinear system

$$
W_{t}(x, t)+\sum_{j=1}^{d} A_{j}(x) \partial_{j} W(x, t)=B(x, W(x, t))+D(W(x, t)),
$$


where $A_{j}(x) \in \mathcal{M}_{N \times N}, j=0, \ldots, d$, and for all nonzero vectors $\xi \in \mathbb{R}^{d}$, the matrix $\sum_{j=1}^{d} \xi_{j} A_{j}(x)$ has real eigenvalues.

The aim of this section is to decouple the full system in order to single out the conserved quantities from the others. We want to motivate the idea of decoupling by considering Carleman's system:

$$
\left\{\begin{array}{l}
f_{1 t}+\frac{1}{\varepsilon} f_{1 x}=\frac{1}{\varepsilon^{2}}\left(f_{2}+f_{1}\right)\left(f_{2}-f_{1}\right), \\
f_{2 t}-\frac{1}{\varepsilon} f_{2 x}=\frac{1}{\varepsilon^{2}}\left(f_{2}+f_{1}\right)\left(f_{1}-f_{2}\right) .
\end{array}\right.
$$

Setting $\rho=f_{1}+f_{2}$ and $m=\frac{f_{1}-f_{2}}{\varepsilon}$ we get that $\rho, m$ satisfy

$$
\left\{\begin{array}{l}
\rho_{t}+m_{x}=0 \\
\varepsilon^{2} m_{t}+\rho_{x}=-2 \rho m
\end{array}\right.
$$

In this way we decoupled (3.2) in (3.3) and we have isolated $\rho$ which represents the conserved quantity. Now we want to do the same procedure on our system (3.1). Let us consider the hypothesis (A.3). Then there exists a matrix $P^{I} \in \mathcal{M}_{k \times N}$ such that $P^{I} B(W)=0$ and $Z^{I}=P^{I} W$ is the conserved vector. Therefore, we can construct an invertible matrix $P=\left[\begin{array}{c}P^{I} \\ P^{I I}\end{array}\right], P^{I I} \in \mathcal{M}_{(N-k) \times N}$. Now, for any $W \in \mathbb{R}^{N}$, we set

$$
\begin{aligned}
& Z^{I}=P^{I} W \\
& Z=\left[\begin{array}{c}
Z^{I} \\
Z^{I I}
\end{array}\right] \\
& Z^{I I}=P^{I I} W, \\
& D^{I}\left(Z^{I}, Z^{I I}\right)=P^{I} D\left(P^{-1} Z\right), \\
& Q\left(Z^{I}, Z^{I I}\right)=P^{I I} B\left(P^{-1} Z\right), \\
& P a(x, \xi) P^{-1}=\left[\begin{array}{ll}
m^{11}(x, \xi) & m^{12}(x, \xi) \\
m^{21}(x, \xi) & m^{22}(x, \xi)
\end{array}\right]=m(x, \xi), \quad M^{i j}(x, D)=O P m^{i j}(x, \xi) ;
\end{aligned}
$$

hence by using the previous notation we can rewrite the system (3.1) in the decoupled form

$$
\left\{\begin{array}{l}
Z_{t}^{I}+M^{11}(x, D) Z^{I}+M^{12}(x, D) Z^{I I}=D^{I}\left(Z^{I}, Z^{I I}\right) \\
Z_{t}^{I I}+M^{21}(x, D) Z^{I}+M^{22}(x, D) Z^{I I}=Q\left(x, Z^{I}, Z^{I I}\right)+D^{I I}\left(Z^{I}, Z^{I I}\right)
\end{array}\right.
$$

where by construction $Z^{I}=Z^{I}(x, t) \in \mathbb{R}^{k}, Z^{I I}=Z^{I I}(x, t) \in \mathbb{R}^{N-k}$ and $m^{11}(x, \xi) \in$ $\mathcal{M}_{k \times k}, m^{12}(x, \xi) \in \mathcal{M}_{k \times(N-k)}, m^{21}(x, \xi) \in \mathcal{M}_{(N-k) \times k}, m^{22}(x, \xi) \in \mathcal{M}_{(N-k) \times(N-k)}$, $D^{I}\left(Z^{I}, Z^{I I}\right) \in \mathbb{R}^{k}, D^{I I}\left(Z^{I}, Z^{I I}\right) \in \mathbb{R}^{N-k}, Q\left(x, Z^{I}, Z^{I I}\right) \in \mathbb{R}^{N-k}$. The previous transformation does not affect the hyperbolic character of our system.

3.2. Structural condition. In order to perform our analysis on system (3.1) we need the following structural assumption:

(S.1) $m^{11}(x, \xi)=0$, for any $(x, \xi) \in \mathbb{R}^{d} \times \mathbb{R}^{d}$. 
This condition is natural if we consider system (3.3). In that case $m(x, \xi)$ is antisymmetric and is given by

$$
m(x, \xi)=\left(\begin{array}{cc}
0 & i \xi \\
i \xi & 0
\end{array}\right)
$$

The same hypothesis is assumed also by Lions and Toscani 33. It was implicitly contained also in the paper of Marcati and Rubino [41. Indeed, in 41] the relaxation of the following quasilinear nonhomogeneous $2 \times 2$ hyperbolic system was taken into consideration:

$$
\left\{\begin{array}{l}
w_{s}+f(w, z)_{y}=0 \\
z_{s}+g(w, z)_{y}=h(w, z),
\end{array}\right.
$$

where $y \in \mathbb{R}, s \geq 0$. In this case the authors required the assumption

$$
f(w, 0)=0,
$$

which in the linear case is equivalent to (S.1). An analogous condition has been assumed by Lattanzio and Yong in [29], where they study the singular limits for the initial value problem

$$
\begin{gathered}
W_{t}+\sum_{j=1}^{d} \bar{A}_{j}(W) W_{x_{j}}+\frac{1}{\varepsilon} \sum_{j=1}^{d} A_{j}(\varepsilon W) W_{x_{j}}=\frac{Q(W)}{\varepsilon^{2}}, \\
W(x, 0)=W_{0}(x ; \varepsilon),
\end{gathered}
$$

by validating the formal asymptotic approximation in the framework of $H^{s}$-smooth solutions. In [29] they set

$$
\bar{A}_{j}=\left(\begin{array}{ll}
\bar{A}_{j}^{11} & \bar{A}_{j}^{12} \\
\bar{A}_{j}^{21} & \bar{A}_{j}^{22}
\end{array}\right), \quad A_{j}=\left(\begin{array}{cc}
A_{j}^{11} & A_{j}^{12} \\
A_{j}^{21} & A_{j}^{22}
\end{array}\right)
$$

and the key structure condition is given by

$$
A_{j}^{11}(0)=0, \quad \text { for all } j .
$$

Therefore, the condition (S.1) is essential; otherwise, the relaxation process would end up to be only the trivial one, relaxing on the null solution. In Appendix A we shall develop formal asymptotic expansions to analyze the situation occurring when (S.1) is violated.

3.3. Block structure. Now, let us consider (3.4). Then, by using the previous structural condition (S.1) and by denoting

$$
M(x, D)=O P\left\{-\left(\begin{array}{cc}
0 & m^{12}(x, \xi) \\
m^{21}(x, \xi) & m^{22}(x, \xi)
\end{array}\right)\right\},
$$

we can rewrite the system (3.4) in the form

$$
Z_{t}-M(x, D) Z=Q\left(x, Z^{I}, Z^{I I}\right)+D\left(Z^{I}, Z^{I I}\right),
$$

where $D\left(Z^{I}, Z^{I I}\right)=\left(D^{I}\left(Z^{I}, Z^{I I}\right), D^{I I}\left(Z^{I}, Z^{I I}\right)\right)$.

We formulate here the hypothesis concerning the existence of a symmetrizer for the system (3.7) in pseudodifferential form (see Taylor [55]), namely: 
(A.4) There exists $R(x, D) \in O P S_{1,0}^{0}$ such that

$$
R(x, D) M(x, D)+(R(x, D) M(x, D))^{*} \in O P S_{1,0}^{0}
$$

and its symbol $r(x, \xi)$ is a positive definite matrix for $|\xi| \geq 1$.

The next structure condition regards the existence of a symmetrizer for the system (3.7). We assume here a special "block structure" which is natural for strictly hyperbolic systems (for instance, see [2] and in the more complicated framework of initial boundary value problems, also see the seminal paper of Kreiss [24], Majda and Osher [36] or Ralston [48]). The block structure follows also for nonstrictly hyperbolic systems having constant multiplicity, by a general result due to Métivier [43. In the sequel we do not assume any one of the previous conditions but directly the "block structure" of the symmetrizer $R(x, D)$.

(S.2) The symbol of $r(x, \xi) \in S_{1,0}^{0}$ of $R(x, D)$ has the form

$$
r(x, \xi)=\left(\begin{array}{cc}
r_{11}(x, \xi) & 0 \\
0 & r_{22}(x, \xi)
\end{array}\right),
$$

where $r_{11}(x, \xi) \in \mathcal{M}_{k \times k}, r_{22}(x, \xi) \in \mathcal{M}_{(N-k) \times(N-k)}$ are symmetric positive definite matrices.

Let us remark that in our examples at the end, these requirements will be easily fulfilled. Let us denote by $m_{1}^{i j}(x, \xi)$ the principal symbol of each block $m^{i j}(x, \xi)$ of the symbol $m(x, \xi)$ (let us remark that $m(x, \xi) \in S^{1}$ is polihomogeneous). By the definition of symmetrizer for the system (3.4) we know the following relations between the blocks of $r(x, \xi)$ and the coefficients of the system (3.4):

$$
\begin{aligned}
& r_{11}(x, \xi) m_{1}^{12}(x, \xi)=-\left(m_{1}^{21}(x, \xi)\right)^{*} r_{22}(x, \xi), \\
& r_{22}(x, \xi) m_{1}^{21}(x, \xi)=-\left(m_{1}^{12}(x, \xi)\right)^{*} r_{11}(x, \xi) .
\end{aligned}
$$

Remark 3.2. The block structure which is assumed in (S.2) implies that the matrix $m_{1}^{12} m_{1}^{21}$ is diagonalizable with real eigenvalues. In fact, $m_{1}^{12} m_{1}^{21}=r_{11}^{-1}\left(m_{1}^{12} m_{1}^{21}\right)^{*} r_{11}$ is the product of two symmetric matrices of which one is positive definite.

Remark 3.3. It is well known that the positivity of symbols does not imply positivity of the operators, but this fact will be true only modulo errors in $O P S^{-\infty}$. By using Theorem 2.2 there exists a pseudodifferential operator $\widetilde{R}(x, D) \in O P S^{1}$, such that $\widetilde{R}(x, D) \geq \eta I>0, \widetilde{R}(x, D)=R(x, D)+T(x, D), T(x, D) \in O P S^{-\infty}$. By the block structure of $R(x, D)$, we can assume a block structure for $T(x, D)$. In a similar way we can approximate fractional powers of $\widetilde{R}(x, D)$ with fractional powers of symbols, modulo errors $T_{\alpha}(x, D) \in O P S^{-\infty}$. Namely, for any $\alpha \in \mathbb{R}$ it follows that $\widetilde{R}^{\alpha}(x, D)=R_{\alpha}(x, D)+T_{\alpha}(x, D)$, where $R_{\alpha}(x, D)=O P\left(r^{\alpha}(x, \xi)\right)$ and $T_{\alpha}(x, D) \in O P S^{-\infty}$.

3.4. Dissipativity condition. In this section we state the assumption on the nonhomogeneous term $Q\left(x, Z^{I}, Z^{I I}\right)$.

(D) $Q\left(x, Z^{I}, Z^{I I}\right) \in C^{1}\left(\mathbb{R}^{N+d} ; \mathbb{R}^{N-k}\right)$ and $Q\left(x, Z^{I}, 0\right)=0$ for any $\left(x, Z^{I}\right) \in$ $\mathbb{R}^{d} \times \mathbb{R}^{k} ;$ moreover,

(d0) $Q\left(x, Z^{I}, Z^{I I}\right)$ has the form

$$
Q\left(x, Z^{I}, Z^{I I}\right)=Q_{0}\left(x, Z^{I}, Z^{I I}\right)+Q_{1}\left(x, Z^{I}, Z^{I I}\right),
$$


(d1) $Q_{0, I I}\left(x, Z^{I}, Z^{I I}\right)$ is bounded in $\left(x, Z^{I}, Z^{I I}\right)$ and there exists $\lambda_{0}>0$ such that for any $\left(x, Z^{I}, Z^{I I}\right) \in \mathbb{R}^{d} \times \mathbb{R}^{k} \times \mathbb{R}^{N-k}, r_{22} Q_{0, I I}\left(x, Z^{I}, Z^{I I}\right)+$ $\left(Q_{0, I I}\left(x, Z^{I}, Z^{I I}\right)\right)^{T} r_{22} \leq-\lambda_{0} r_{22}$,

(d2) $Q_{1, I I}\left(x, Z^{I}, Z^{I I}\right)$ is bounded in $\left(x, Z^{I}, Z^{I I}\right)$ and the operator $\left(R_{22}\right)_{1 / 2} Q_{1, I I}\left(R_{22}\right)_{-1 / 2}$ satisfies $\left\|\left(R_{22}\right)_{1 / 2} Q_{1, I I}\left(R_{22}\right)_{-1 / 2}\right\|_{\mathcal{L}\left(L^{2}\right)} \leq \lambda_{1}$, $\lambda_{1}>0, \lambda_{1} \leq \lambda_{0} / 2$; moreover, $\left\|Q_{0, I I}^{-1} Q_{1, I I}\right\|_{\mathcal{L}\left(L^{2}\right)} \leq \gamma<1$,

where $\left(R_{22}\right)_{1 / 2}$ is defined as in Remark 3.3

Remark 3.4. We divided the nonhomogeneous term $Q\left(x, Z^{I}, Z^{I I}\right)$ into two terms that take into account different dissipativity mechanisms of $Q$. The former term $Q_{0}\left(x, Z^{I}, Z^{I I}\right)$ is related to the dissipativity of $Q$, the latter $Q_{1, I I}\left(x, Z^{I}, Z^{I I}\right)$ defines weighted by with $\left(R_{22}\right)_{1 / 2}$ and $\left(R_{22}\right)_{-1 / 2}$ a bounded operator dominated by the dissipative part of $Q$. The assumptions made in (d1), (d2) are scale invariant.

Remark 3.5. In many applications it could be useful to take $Q$ in the form

$$
Q\left(x, Z^{I}, Z^{I I}\right)=C(x) Z^{I I},
$$

where for any $x, \xi \in \mathbb{R}^{d}, C(x) \in \mathcal{M}_{(N-k) \times(N-k)},\left[r_{22}(x, \xi), C(x)\right]=0$ and there exists $\lambda>0$ such that $C(x) \leq-\lambda I$.

Remark 3.6. (i) By using Remark 3.3 and Theorem 2.2, we get that

$$
\widetilde{R}_{22}(x, D) Q_{0, I I}\left(x, Z^{I}, Z^{I I}\right)+\left(Q_{0, I I}\left(x, Z^{I}, Z^{I I}\right)\right)^{T} \widetilde{R}_{22}(x, D) \leq-\lambda_{0} \widetilde{R}_{22}(x, D),
$$

modulo $O P S^{-\infty}$.

(ii) The hypothesis $(\mathrm{d} 2),\left\|Q_{0, I I}^{-1} Q_{1, I I}\right\|_{\mathcal{L}(L 2)} \leq \gamma<1$, implies $Q_{I I}$ is invertible. In particular, $Q_{I I}^{-1}$ has the following form:

$$
Q_{I I}^{-1}=Q_{0, I I}^{-1}+q \text { where } \quad\|q\|_{\infty} \leq \frac{\gamma\left\|Q_{0, I I}^{-1}\right\|_{\infty}}{1-\gamma}
$$

3.5. Formal analysis of the singular limit. We will analyze the relaxation process of the system

$$
W_{t}+\frac{1}{\varepsilon} A(x, D) W=\frac{1}{\varepsilon^{2}} B(x, W)+\frac{1}{\varepsilon} D(W) .
$$

Following the constructions of the previous paragraph we rewrite (3.10), hence

$$
\left\{\begin{aligned}
Z_{t}^{I}+ & \frac{1}{\varepsilon} M^{12}(x, D) Z^{I I}=\frac{1}{\varepsilon} D^{I}\left(Z^{I}, Z^{I I}\right), \\
Z_{t}^{I I} & +\frac{1}{\varepsilon} M^{21}(x, D) Z^{I}+\frac{1}{\varepsilon} M^{22}(x, D) Z^{I I} \\
& =\frac{1}{\varepsilon^{2}} Q\left(x, Z^{I}, Z^{I I}\right)+\frac{1}{\varepsilon} D^{I I}\left(Z^{I}, Z^{I I}\right) .
\end{aligned}\right.
$$

By formal asymptotics we are led to define

$$
Z^{I}(x, t)=U^{I}(x, t), \quad Z^{I I}(x, t)=\varepsilon U^{I I}(x, t) .
$$

The previous scaling could also be obtained from scaling the time variable such that $\partial_{\tau}=\varepsilon \partial_{t}$; for more details see [41]. The system (3.11) under the change of 
variables (3.12) becomes

$$
\left\{\begin{array}{c}
U_{t}^{I}+M^{12}(x, D) U^{I I}=\frac{1}{\varepsilon} D^{I}\left(U^{I}, \varepsilon U^{I I}\right) \\
\varepsilon^{2} U_{t}^{I I}+M^{21}(x, D) U^{I}+\varepsilon M^{22}(x, D) U^{I I} \\
=\frac{1}{\varepsilon} Q\left(x, U^{I}, \varepsilon U^{I I}\right)+D^{I I}\left(U^{I}, \varepsilon U^{I I}\right) .
\end{array}\right.
$$

If we denote by $\left(U^{I 0}, U^{I I 0}\right)$ the limit profile as $\varepsilon \downarrow 0$, the formal limit system (3.13) relaxes to the system

$$
\left\{\begin{array}{l}
U_{t}^{I 0}+M^{12}(x, D) U^{I I 0}=D_{I I}^{I}\left(U^{I 0}, 0\right) U^{I I 0} \\
M^{21}(x, D) U^{I 0}=Q_{I I}\left(x, U^{I 0}, 0\right) U^{I I 0}+D^{I I}\left(U^{I 0}, 0\right) .
\end{array}\right.
$$

By using point (ii) of Remark 3.6 the system (3.14) transforms into

$$
\left\{\begin{array}{l}
U_{t}^{I 0}+M^{12}(x, D) U^{I I 0}=D_{I I}^{I}\left(U^{I 0}, 0\right) U^{I I 0} \\
U^{I I 0}=Q_{I I}^{-1}\left(x, U^{I 0}, 0\right)\left[M^{21}(x, D) U^{I 0}-D^{I I}\left(U^{I 0}, 0\right)\right] .
\end{array}\right.
$$

That is equivalent to (by setting $U=U^{I 0}$ ) the second-order parabolic system

$$
\begin{aligned}
U_{t} & +M^{12}(x, D) Q_{I I}^{-1}(x, U, 0) M^{21}(x, D) U=M^{12}(x, D) Q_{I I}^{-1}(x, U, 0) D^{I I}(U, 0) \\
& +D_{I I}^{I}(U, 0) Q_{I I}^{-1}(x, U, 0)\left[M^{21}(x, D) U-D^{I I}(U, 0)\right] .
\end{aligned}
$$

In the next section we provide sufficient conditions in order to rigorously justify this formal analysis.

3.6. Parabolicity condition. Let us restrict our attention to the differential operator case, namely (1.1) takes the form

$$
W_{t}+\frac{1}{\varepsilon} \sum_{j=1}^{d} A_{j}(x) \partial_{j} W=\frac{1}{\varepsilon^{2}} B(x, W)+\frac{1}{\varepsilon} D(W) .
$$

In this case the identities (3.14) become

$$
\left\{\begin{array}{l}
U_{t}^{I 0}+\sum_{j=1}^{d} M_{j}^{12}(x) \partial_{j} U^{I I 0}=D_{I I}^{I}\left(U^{I 0}, 0\right) U^{I I 0} \\
\sum_{j=1}^{d} M_{j}^{21}(x) \partial_{j} U^{I 0}=Q_{I I}\left(x, U^{I 0}, 0\right) U^{I I 0}+D^{I I}\left(U^{I 0}, 0\right),
\end{array}\right.
$$

which is equivalent to (where we set $U=U^{I 0}$ )

$$
\begin{aligned}
U_{t} & +\sum_{j=1}^{d} M_{j}^{12}(x) \partial_{j}\left(Q_{I I}^{-1}(x, U, 0) \sum_{k=1}^{d} M_{k}^{21}(x) \partial_{k} U\right) \\
& =\sum_{j=1}^{d} M_{j}^{12}(x) \partial_{j}\left(Q_{I I}^{-1}(x, U, 0) D^{I I}(U, 0)\right) \\
& +D_{I I}^{I}(U, 0) Q_{I I}^{-1}(x, U, 0)\left[\sum_{k=1}^{d} M_{k}^{21}(x) \partial_{k} U-D^{I I}(U, 0)\right] .
\end{aligned}
$$


We want to show, in this simpler case, that (3.17) is parabolic in the sense of Petrowski as recalled in Section 2. Taking into account the notation of Section 2, one has

$$
L=-m^{12}(x, \xi) Q_{I I}^{-1}(x, U, 0) m^{21}(x, \xi) \in S_{1,0}^{2}
$$

where

$$
\begin{aligned}
& m^{12}(x, \xi)=m_{1}^{12}(x, \xi)=i \sum_{j=1}^{d} M_{j}^{12}(x) \xi_{j}, \\
& m^{21}(x, \xi)=m_{1}^{21}(x, \xi)=i \sum_{j=1}^{d} M_{j}^{12}(x) \xi_{j} .
\end{aligned}
$$

We are going to show the existence of a positive matrix $P_{0}$ such that $P_{0} L+L^{*} P_{0}$ is negative definite. In the next result we will show that in general $P_{0} L+L^{*} P_{0}$ is positive semidefinite and it is negative definite under the condition that $m^{21}$ is injective. This is related to the Kawashima type condition $(\mathrm{K})$ discussed in Section 4.2. Taking into account the assumption $\left\|Q_{0, I I}^{-1} Q_{1, I I}\right\| \leq \gamma<1$ and Remark [3.6] we have the following theorem.

Theorem 3.7. Assume $0<\gamma<1 /\left(2 \lambda_{0}\left\|Q_{0, I I}^{-1}\right\|+1\right)$. Then there exists $\beta_{0}>0$ such that

$$
\left(\left(P_{0} L+L^{*} P_{0}\right) \eta, \eta\right) \leq-\beta_{0}\left|m^{21} \eta\right|^{2}, \quad \text { for all } \eta \in \mathbb{R}^{k} .
$$

Moreover, if we assume $m^{21}$ is an injective map, then (3.17) is parabolic in the sense of Petrowski; namely, there exists $\beta_{1}>0$ such that

$$
\left(\left(P_{0} L+L^{*} P_{0}\right) \eta, \eta\right) \leq-\beta_{1}|\eta|^{2}, \quad \text { for all } \eta \in \mathbb{R}^{k} .
$$

Proof. We write

$$
P_{0}=r_{11}(x, \xi) .
$$

By using the relations (3.8) and (ii) of Remark 3.6 it follows that

$$
\begin{aligned}
\left(\left(P_{0} L+L^{*} P_{0}\right) \eta, \eta\right) & =-\left(r_{11} m^{12} Q_{I I}^{-1}(x, U, 0) m^{21} \eta, \eta\right) \\
& -\left(\left(m^{21}\right)^{*}\left(Q_{I I}^{-1}(x, U, 0)\right)^{T}\left(m^{12}\right)^{*} r_{11} \eta, \eta\right) \\
& =\left(\left(m^{21}\right)^{*} r_{22} Q_{I I}^{-1}(x, U, 0) \eta, \eta\right) \\
& +\left(\left(m^{21}\right)^{*}\left(\left(Q_{I I}^{-1}(x, U, 0)\right)^{T} r_{22}\right) m^{21} \eta, \eta\right) \\
& =\left(\left(r_{22} Q_{I I}^{-1}(x, U, 0)+\left(Q_{I I}^{-1}(x, U, 0)\right)^{T} r_{22}\right) m^{21} \eta, m^{21} \eta\right) \\
& =\left(\left(r_{22} Q_{0, I I}^{-1}(x, U, 0)+\left(Q_{0, I I}^{-1}(x, U, 0)\right)^{T} r_{22}\right) m^{21} \eta, m^{21} \eta\right) \\
& +\left(\left(r_{22} q+q^{T} r_{22}\right) m^{21} \eta, m^{21} \eta\right) \\
& =J_{1}+J_{2} .
\end{aligned}
$$

By the dissipative condition (d1) we get

$$
\begin{aligned}
J_{1} & =\left(\left(r_{22} Q_{0, I I}^{-1}(x, U, 0)+\left(Q_{0, I I}^{-1}(x, U, 0)\right)^{T} r_{22}\right) m^{21} \eta, m^{21} \eta\right) \\
& \leq\left(\left(r_{22}^{1 / 2} Q_{0, I I}^{-1}(x, U, 0) r_{22}^{-1 / 2}+r_{22}^{1 / 2}\left(Q_{0, I I}^{-1}(x, U, 0)\right)^{T} r_{22}^{-1 / 2}\right) r_{22}^{1 / 2} m^{21} \eta, r_{22}^{1 / 2} m^{21} \eta\right) \\
& \leq-\frac{k_{0}}{\lambda_{0}}\left|m^{21} \eta\right|^{2} .
\end{aligned}
$$


Condition (ii) of Remark 3.6 leads to

$$
J_{2}=\left(\left(r_{22} q+q^{T} r_{22}\right) m^{21} \eta, m^{21} \eta\right) \leq 2 k_{0} \frac{\left\|Q_{0, I I}^{-1}\right\| \gamma}{1-\gamma}\left|m^{21} \eta\right|^{2} .
$$

By using the condition $\gamma<1 /\left(2 \lambda_{0}\left\|Q_{0, I I}^{-1}\right\|+1\right)$ it follows that

$$
\left(\left(P_{0} L+L^{*} P_{0}\right) \eta, \eta\right)=J_{1}+J_{2} \leq-\frac{k_{0}}{2 \lambda_{0}}\left|m^{21} \eta\right|^{2} .
$$

\section{A priori estimates AND CONVERGENCE ANALYsis}

In this section we consider the rescaled system

$$
\left\{\begin{array}{c}
U_{t}^{I}+M^{12}(x, D) U^{I I}=\frac{1}{\varepsilon} D^{I}\left(U^{I}, \varepsilon U^{I I}\right), \\
\varepsilon^{2} U_{t}^{I I}+M^{21}(x, D) U^{I}+\varepsilon M^{22}(x, D) U^{I I} \\
\quad=\frac{1}{\varepsilon} Q\left(x, U^{I}, \varepsilon U^{I I}\right)+D^{I I}\left(U^{I}, \varepsilon U^{I I}\right),
\end{array}\right.
$$

and we are going to develop a rigorous theory in order to converge to the relaxed system (3.14). We want to show that, as $\varepsilon \downarrow 0$, the solutions of the rescaled system satisfy

$$
\begin{aligned}
& U^{I} \longrightarrow U^{I 0} \quad \text { strongly in }\left[L_{\text {loc }}^{2}\left(\mathbb{R}^{d} \times \mathbb{R}_{+}\right)\right]^{k}, \\
& U^{I I} \rightarrow U^{I I 0} \quad \text { weakly in }\left[L^{2}\left(\mathbb{R}^{d} \times[0, T]\right)\right]^{N-k}, \\
& \varepsilon U^{I I} \longrightarrow 0 \quad \text { strongly in }\left[L_{\text {loc }}^{2}\left(\mathbb{R}^{d} \times \mathbb{R}_{+}\right)\right]^{N-k} \text {. }
\end{aligned}
$$

The basic idea used in this section is the assumption of the existence of a symmetrizer for the system (3.4), whose symbol has a suitable block structure (conditions (A.4) and (S.2)). The properties of the symmetrizer (see, for instance, Theorem 2.1 and Theorem 2.2) allow us to establish "energy" type estimates.

4.1. A priori estimates. In this section we wish to establish a priori estimates, independent of $\varepsilon$, for the solution of the system (4.1). To achieve this goal the following hypotheses are needed:

(B.1) $m^{12}(x, \xi), m^{21}(x, \xi), m^{22}(x, \xi) \in S^{1}$,

(B.2) $D=\left(D^{I}\left(Z^{I}, Z^{I I}\right), D^{I I}\left(Z^{I}, Z^{I I}\right)\right) \in C^{1}\left(\mathbb{R}^{N} ; \mathbb{R}^{k} \times \mathbb{R}^{N-k}\right), D^{I}\left(Z^{I}, 0\right)=0$, $D^{I I}(0,0)=0$, and $D_{I I}^{I}$ is bounded in $\left(Z^{I}, Z^{I I}\right), D^{I I}$ is a lipschitz function in $\left(Z^{I}, Z^{I I}\right)$, with lipschitz norm $\alpha$.

Remark 4.1. We could weaken our assumptions concerning the regularity of the coefficients by using directly the Bony paradifferential calculus ([56], Section 3.4) but we want to avoid additional technical complications.

The next result concerns the "energy" type estimates independent on $\varepsilon$, obtained via the existence of symmetrizers and via the dissipativity conditions (D).

Theorem 4.2. Let us consider the solution $\left\{U^{I}\right\},\left\{U^{I I}\right\}$ of the Cauchy problem for the scaled system (4.1). Assume $U^{I}(x, 0) \in\left[L^{2}\left(\mathbb{R}^{d}\right)\right]^{k}, U^{I I}(x, 0) \in\left[L^{2}\left(\mathbb{R}^{d}\right)\right]^{N-k}$ and hypotheses (A.4), (S.2), (B.1), (B.2), (D) hold. Then, there exists $\varepsilon_{0}>0$, such that for $\varepsilon \in\left(0, \varepsilon_{0}\right)$, one has

(i) for all $T>0$, there exists $M(T)>0$, independent of $\varepsilon$, such that $\left\|U^{I I}\right\|_{L^{2}\left(\mathbb{R}^{d} \times[0, T]\right)} \leq M(T)$ and $\sup _{[0, T]}\left\|\varepsilon U^{I I}(\cdot, t)\right\| \leq M(T)$, 
(ii) $\left\{\varepsilon^{2} U_{t}^{I I}\right\}$ is relatively compact in $H_{\text {loc }}^{-1}\left(\mathbb{R}^{d} \times \mathbb{R}_{+}\right)$,

(iii) $\left\{U^{I}\right\}$ is uniformly bounded, with respect to $\varepsilon$, in $L^{\infty}\left(\mathbb{R}_{+}, L^{2}\left(\mathbb{R}^{d}\right)\right)$, namely for all $T>0$, there exists $M(T)>0$, independent of $\varepsilon$, such that $\sup _{[0, T]}\left\|U^{I}(\cdot, t)\right\| \leq M(T)$.

Proof. To simplify our notation, we set

$$
\begin{gathered}
U=\left[\begin{array}{c}
U^{I} \\
U^{I I}
\end{array}\right], \quad U^{\varepsilon}=\left[\begin{array}{c}
U^{I} \\
\varepsilon U^{I I}
\end{array}\right], \quad F=\left[\begin{array}{c}
\frac{1}{\varepsilon} D^{I}\left(U^{I}, \varepsilon U^{I I}\right) \\
\frac{1}{\varepsilon} Q\left(x, U^{I}, \varepsilon U^{I I}\right)+D^{I I}\left(U^{I}, \varepsilon U^{I I}\right)
\end{array}\right], \\
M^{\varepsilon}(x, D)=O P\left\{-\left(\begin{array}{cc}
0 & m^{12}(x, \xi) \\
m^{21}(x, \xi) & \varepsilon m^{22}(x, \xi)
\end{array}\right)\right\} .
\end{gathered}
$$

Let $R(x, D)$ be the symmetrizer given by hypotheses (A.4), (S.2), by using Theorem 2.2. Then there exists $\widetilde{R}=R+T, T=O P(t(x, \xi)) \in O P S^{-\infty}$, with $\widetilde{R} \geq \eta I>0$. By the block structure of $R(x, D)$, we can assume that the symbol $t(x, \xi)$ takes the form $t(x, \xi)=\left(\begin{array}{cc}t_{11}(x, \xi) & 0 \\ 0 & t_{22}(x, \xi)\end{array}\right)$. Hence it follows that

$$
\begin{aligned}
\frac{d}{d t}\left(\widetilde{R}(x, D) U^{\varepsilon}, U^{\varepsilon}\right)_{2}= & \left(\widetilde{R}(x, D) U_{t}^{\varepsilon}, U^{\varepsilon}\right)_{2}+\left(\widetilde{R}(x, D) U^{\varepsilon}, U_{t}^{\varepsilon}\right)_{2} \\
= & \left(\widetilde{R}(x, D)\left(M^{\varepsilon}(x, D) U+F\right), U\right)_{2}+\left(\widetilde{R}(x, D) U, M^{\varepsilon}(x, D) U+F\right)_{2} \\
= & \left(\left(\widetilde{R}(x, D) M^{\varepsilon}(x, D)+\left(\widetilde{R}(x, D) M^{\varepsilon}(x, D)\right)^{*}\right) U, U\right)_{2} \\
& +2(\widetilde{R}(x, D) F, U)_{2}=I_{1}+I_{2} .
\end{aligned}
$$

We are going to estimate separately $I_{1}$ and $I_{2}$. By applying Theorem [2.1] and Theorem 2.2, and by using hypothesis (A.4) for $I_{1}$ we get

$$
I_{1} \leq 2 c_{0}\left\|U^{I I}\right\|\left\|U^{I}\right\|+\varepsilon c_{0}\left\|U^{I I}\right\|^{2},
$$

where $c_{0}=\left\|\widetilde{R}(x, D) M^{\varepsilon}(x, D)+\left(\widetilde{R}(x, D) M^{\varepsilon}(x, D)\right)^{*}\right\|_{\mathcal{L}\left(L^{2}\right)}$. Let us focus our attention on $I_{2}$. Then one has

$$
\begin{aligned}
I_{2}= & \left(\widetilde{R}(x, D) D\left(U^{I}, \varepsilon U^{I I}\right), U\right)_{2}+\left(\widetilde{R}(x, D)\left(\begin{array}{c}
0 \\
\varepsilon^{-1} Q\left(x, U^{I}, \varepsilon U^{I I}\right)
\end{array}\right), U\right)_{2} \\
= & \left(\varepsilon^{-1} D^{I}\left(U^{I}, \varepsilon U^{I I}\right), \widetilde{R}_{11}(x, D) U^{I}\right)_{2}+\left(D^{I I}\left(U^{I}, \varepsilon U^{I I}\right), \widetilde{R}_{22}(x, D) U^{I I}\right)_{2} \\
& +\left(\varepsilon^{-1} \widetilde{R}_{22}(x, D) Q\left(x, U^{I}, \varepsilon U^{I I}\right), U^{I I}\right)_{2}=I_{2,1}+I_{2,2}+I_{2,3} .
\end{aligned}
$$

To estimate $I_{2,1}$ and $I_{2,2}$ we use hypothesis (B.2) and we get

$$
I_{2,1}+I_{2,2} \leq \alpha\left(c_{1}+c_{2}\right)\left\|U^{I I}\right\|\left\|U^{I}\right\|+\alpha \varepsilon c_{2}\left\|U^{I I}\right\|^{2},
$$

where $c_{1}=\left\|\widetilde{R}_{11}\right\|_{\mathcal{L}\left(L^{2}\right)}, c_{2}=\left\|\widetilde{R}_{22}\right\|_{\mathcal{L}\left(L^{2}\right)}$. Now we turn to $I_{2,3}$. Let us set $\bar{Q}_{I I}=$ $\int_{0}^{1} Q_{I I}\left(x, U^{I}, \varepsilon \theta U^{I I}\right) d \theta$; then we can rewrite $I_{2,3}$ in the following way:

$$
I_{2,3}=\left(\widetilde{R}_{22}(x, D) \bar{Q}_{0, I I} U^{I I}, U^{I I}\right)_{2}+\left(\widetilde{R}_{22}(x, D) \bar{Q}_{1, I I} U^{I I}, U^{I I}\right)_{2}=I_{2,31}+I_{2,32} .
$$

From (d1) and Remark 3.6, it follows that

$$
\begin{aligned}
I_{2,31} & =\left(\left(\widetilde{R}_{22}(x, D) \bar{Q}_{0, I I}+\left(\bar{Q}_{0, I I}\right)^{T} \widetilde{R}_{22}(x, D)\right) U^{I I}, U^{I I}\right)_{2} \\
& \leq-\lambda_{0} c_{3}^{2}\left\|U^{I I}\right\|^{2}+c_{4}\left\|U^{I I}\right\|_{H^{-1}}\left\|U^{I I}\right\|,
\end{aligned}
$$


where $c_{3}=\left\|\widetilde{R}_{22}^{1 / 2}\right\|_{\mathcal{L}\left(L^{2}\right)}$. By using (d2) we get

$$
\begin{aligned}
I_{2,32} & =\left(\widetilde{R}_{22} \bar{Q}_{1, I I} \widetilde{R}_{22}^{-1 / 2} \widetilde{R}_{22}^{1 / 2} U^{I I}, \widetilde{R}_{22}^{-1 / 2} \widetilde{R}_{22}^{1 / 2} U^{I I}\right)_{2} \\
& =\left(\left(R_{22}\right)_{1 / 2} \bar{Q}_{1, I I}\left(R_{22}\right)_{-1 / 2} \widetilde{R}_{22}^{1 / 2} U^{I I} \widetilde{R}_{22}^{1 / 2} U^{I I}\right)_{2}+\left(\bar{T}_{22} U^{I I}, \widetilde{R}_{22}^{1 / 2} U^{I I}\right)_{2} \\
& \leq \lambda_{1} c_{3}^{2}\left\|U^{I I}\right\|^{2}+c_{3} c_{5}\left\|U^{I I}\right\|_{H^{-1}}\left\|U^{I I}\right\| \leq \frac{\lambda_{0}}{2} c_{3}\left\|U^{I I}\right\|^{2}+c_{3} c_{5}\left\|U^{I I}\right\|_{H^{-1}}\left\|U^{I I}\right\|,
\end{aligned}
$$

where

$$
\begin{aligned}
\bar{T}_{22}= & \left(R_{22}\right)_{1 / 2}\left(\left(R_{22}\right)_{-1 / 2}+\left(T_{22}\right)_{-1 / 2}\right)\left(R_{22}\right)_{1 / 2} \\
& +\left(T_{22}\right)_{1 / 2}\left(\left(R_{22}\right)_{-1 / 2}+\left(T_{22}\right)_{-1 / 2}\right)\left(R_{22}\right)_{1 / 2}
\end{aligned}
$$

and $c_{5}=\left\|\bar{T}_{22}\right\|_{\mathcal{L}\left(H^{-1}, L^{2}\right)}$. Hence

$$
I_{2,3}=-\frac{\lambda_{0}}{2} c_{3}^{2}\left\|U^{I I}\right\|^{2}+\left(c_{3} c_{5}+c_{4}\right)\left\|U^{I I}\right\|_{H^{-1}}\left\|U^{I I}\right\|,
$$

and for any $\delta_{1}>0$ we have

$$
I_{2,3} \leq-\frac{\lambda_{0}}{2} c_{3}^{2}\left\|U^{I I}\right\|^{2}+\left(c_{3} c_{5}+c_{4}\right) \delta_{1}\left\|U^{I I}\right\|^{2}+\frac{c_{3} c_{5}+c_{4}}{\delta_{1}}\left\|U^{I I}\right\|_{H^{-1}}^{2} .
$$

By adding $I_{1}, I_{2}$, for all $\delta_{1}>0, \delta_{2}>0$, we get

$$
\begin{aligned}
\frac{d}{d t}\left(\widetilde{R}(x, D) U^{\varepsilon}, U^{\varepsilon}\right)_{2} \leq & \left(2 c_{0}+\alpha\left(c_{1}+c_{2}\right)\right)\left\|U^{I I}\right\|\left\|U^{I}\right\|+\varepsilon\left(c_{0}+\alpha c_{2}\right)\left\|U^{I I}\right\|^{2} \\
& -\frac{\lambda_{0}}{2} c_{3}^{2}\left\|U^{I I}\right\|^{2}+\left(c_{3} c_{5}+c_{4}\right) \delta_{1}\left\|U^{I I}\right\|^{2}+\frac{c_{3} c_{5}+c_{4}}{\delta_{1}}\left\|U^{I I}\right\|_{H^{-1}}^{2} \\
\leq & K\left(\delta_{1}+\delta_{2}\right)\left\|U^{I I}\right\|^{2}-\frac{\lambda_{0}}{2}\left\|U^{I I}\right\|^{2}+\frac{K}{\delta_{2}}\left\|U^{I}\right\|^{2} \\
& +\frac{K}{\delta_{1}}\left\|U^{I I}\right\|_{H^{-1}}^{2} \varepsilon K\left\|U^{I I}\right\|^{2},
\end{aligned}
$$

where $K=K\left(c_{0}, c_{1}, c_{2}, c_{3}, c_{4}, c_{5}, \alpha\right)$. If we choose $\varepsilon<\frac{\lambda_{0}}{8}$ and $\left(\delta_{1}+\delta_{2}\right)<\frac{\lambda_{0}}{8}$, it follows that

$$
\frac{d}{d t}\left(\widetilde{R}(x, D) U^{\varepsilon}, U^{\varepsilon}\right)_{2} \leq-\frac{\lambda_{0}}{4} K\left\|U^{I I}\right\|^{2}+\frac{K}{\delta_{2}}\left\|U^{I}\right\|^{2}+\frac{K}{\delta_{1}}\left\|U^{I I}\right\|_{H^{-1}}^{2} .
$$

Let

$$
E(t)=\int_{\mathbb{R}^{d}} \varepsilon^{2}\left|U^{I I}(x, t)\right|^{2} d x+\int_{\mathbb{R}^{d}}\left|U^{I}(x, t)\right|^{2} d x .
$$

By the second equation of the system (4.1) we get the estimate

$$
\left\|U^{I I}\right\|_{H^{-1}}^{2} \leq K E(t) .
$$

By integrating (4.2) on $[0, t]$ we obtain that the energy $E(t)$ satisfies the integral inequality

$$
E(t) \leq E(0)-\frac{\lambda_{0}}{4} \int_{0}^{t} \int_{\mathbb{R}^{d}}\left|U^{I I}(x, s)\right|^{2} d x d s+K \int_{0}^{t} E(s) d s,
$$

for some $c>0$. Then by applying Gronwall's lemma, we have

$$
E(t) \leq E(0) e^{K t}
$$


and

$$
\int_{0}^{t} \int_{\mathbb{R}^{d}}\left|U^{I I}\right|^{2} d x d s \leq \frac{4 K}{\lambda_{0}} E(0) e^{K t} .
$$

Hence we conclude that for any $T>0$ there exists $M(T)>0$, independent of $\varepsilon$, such that

$$
\left\|U^{I I}\right\|_{L^{2}\left(\mathbb{R}^{d} \times[0, T]\right)} \leq M(T), \quad \sup _{[0, T]}\left\|U^{I I}(\cdot, t)\right\| \leq M(T), \quad \sup _{[0, T]}\left\|U^{I}(\cdot, t)\right\| \leq M(T) .
$$

In this way we have proved (i) and (iii). Let us consider $\omega$ as a relatively compact subset of $\mathbb{R}^{d} \times \mathbb{R}_{+}$. Then

$$
\begin{aligned}
\left\|\varepsilon^{2} U_{t}^{I I}\right\|_{H^{-1}(\omega)} & =\sup _{\|\phi\|_{H_{0}^{1}(\omega)}=1}\left|\left\langle\varepsilon^{2} U_{t}^{I I}, \phi\right\rangle\right|=\sup _{\|\phi\|_{H_{0}^{1}(\omega)}=1}\left|\iint \varepsilon^{2} U^{I I} \phi_{t} d x d t\right| \\
& \leq \varepsilon^{2} \sup _{\|\phi\|_{H_{0}^{1}(\omega)}=1}\left(\left\|U^{I I}\right\|_{L^{2}(\omega)}\left\|\phi_{t}\right\|_{L^{2}(\omega)}\right) \leq M(T) \varepsilon^{2} .
\end{aligned}
$$

Remark 4.3. If, in an independent way, we know a bound for $\left\|Q_{I I}\right\|_{L i p}$, we do not need the assumptions (D); but to estimate $I_{2,3}$ it is sufficient, for instance, to have, for any $\left(x, Z^{I}, Z^{I I}\right) \in \mathbb{R}^{d} \times \mathbb{R}^{k} \times \mathbb{R}^{N-k}, Q_{I I}\left(x, Z^{I}, Z^{I I}\right) \leq-\lambda I, \lambda>0$. Indeed,

$$
\begin{aligned}
I_{2,3} & =\left(\widetilde{R}_{22}^{1 / 2} \bar{Q}_{I I} U^{I I}, \widetilde{R}_{22}^{1 / 2} U^{I I}\right)_{2} \\
& =\left(\bar{Q}_{I I} \widetilde{R}_{22}^{1 / 2} U^{I I}, \widetilde{R}_{22}^{1 / 2} U^{I I}\right)_{2}+\left(\left[\widetilde{R}_{22}^{1 / 2}, \bar{Q}_{I I}\right] U^{I I}, U^{I I}\right)_{2} .
\end{aligned}
$$

Then by taking into account Proposition 2.3 and the dissipative condition on $Q_{I I}\left(x, U^{I}, \varepsilon U^{I I}\right)$, we have

$$
I_{2,3} \leq-\lambda\left\|U^{I I}\right\|^{2}+K\left\|Q_{I I}\right\|_{L i p}\left\|U^{I I}\right\|_{H^{-1}}^{2},
$$

which leads to the energy inequality (4.4). Namely, in this case the commutator $\left[\widetilde{R}_{22}^{1 / 2}, \bar{Q}_{I I}\right]$ regularizes enough to close the estimates.

4.2. Strong convergence analysis. In order to get the convergence we will introduce a Kawashima [22] type condition (K) similar to the assumption (G.5), Section 6.2 in 41 and to the assumption in Lemma 2.4 in [29]. Let us denote by $m_{1}^{i j}(x, \xi)$ the principal symbol of each block $m^{i j}(x, \xi)$ of the symbol $m(x, \xi)$ (let us remark that $m(x, \xi) \in S^{1}$ is polihomogeneous); we assume

$$
(\mathbf{K}) \operatorname{det}\left[\left(m_{1}^{21}(x, \xi)\right)^{*} m_{1}^{21}(x, \xi)\right] \neq 0 \text {. }
$$

Remark 4.4. Since $m^{21}(x, \xi) \in \mathcal{M}_{(N-k) \times k}$, from elementary linear algebra we deduce condition (K) is violated whenever $k>\frac{N}{2}$.

Remark 4.5. Condition $(\mathrm{K})$ is actually equivalent to the Kawashima condition, namely that $\operatorname{rank}\left(m_{1}^{12}(x, \xi)\right)=k$. Indeed, condition $(\mathrm{K})$ means that $m_{1}^{21}(x, \xi)$ is injective, namely that its transpose is onto. From the relations (3.8), we obtain the equivalent property that $m_{1}^{12}(x, \xi)$ is onto, namely that $\operatorname{rank}\left(m_{1}^{12}(x, \xi)\right)=k$.

We begin with an immediate consequence of (i) and (ii) in Theorem (4.2). 
Theorem 4.6. Let us consider the solution $\left\{U^{I I}\right\}$ of the Cauchy problem for system (4.1). Assume $U^{I}(x, 0) \in\left[L^{2}\left(\mathbb{R}^{d}\right)\right]^{k}, U^{I I}(x, 0) \in\left[L^{2}\left(\mathbb{R}^{d}\right)\right]^{N-k}$ and, moreover, the hypotheses (A.4), (S.2), (B.1), (B.2), (D) hold. Then there exists $U^{I I 0} \in$ $\left[L^{2}\left(\mathbb{R}^{d} \times[0, T]\right)\right]^{N-k}$ such that, as $\varepsilon \downarrow 0$, one has (extracting eventually a subsequence)

$$
\begin{array}{ll}
U^{I I} \rightarrow U^{I I 0} & \text { weakly in }\left[L^{2}\left(\mathbb{R}^{d} \times[0, T]\right)\right]^{N-k}, \\
\varepsilon U^{I I} \longrightarrow 0 & \text { strongly in }\left[L_{\text {loc }}^{2}\left(\mathbb{R}^{d} \times \mathbb{R}_{+}\right)\right]^{k}, \\
\left\{\varepsilon^{2} U_{t}^{I I}\right\} \longrightarrow 0 & \text { in }\left[H_{\text {loc }}^{-1}\left(\mathbb{R}^{d} \times \mathbb{R}_{+}\right)\right]^{N-k} .
\end{array}
$$

Our next step is to prove the strong convergence for the sequence $\left\{U^{I}\right\}$ in the norm of $L_{\text {loc }}^{2}\left(\mathbb{R}^{d} \times \mathbb{R}_{+}\right)$. For this purpose we only use the estimates obtained in the previous section. Our main tool in the limit process will be Tartar's and Gérard's Theorem 2.4 (54], 13]).

Theorem 4.7. Let us consider the solution $\left\{U^{I}\right\}$ of the Cauchy problem for system (4.11). Assume $U^{I}(x, 0) \in\left[L^{2}\left(\mathbb{R}^{d}\right)\right]^{k}, U^{I I}(x, 0) \in\left[L^{2}\left(\mathbb{R}^{d}\right)\right]^{N-k}$ and the hypotheses (A.4), (S.2), (B.1), (B.2), (K), (D) hold. Then there exists $U^{I 0} \in\left[L^{2}\left(\mathbb{R}^{d} \times[0, T]\right)\right]^{k}$ such that, as $\varepsilon \downarrow 0$, one has (extracting eventually subsequences)

$$
U^{I} \longrightarrow U^{I 0} \quad \text { strongly in }\left[L_{\text {loc }}^{2}\left(\mathbb{R}^{d} \times \mathbb{R}_{+}\right)\right]^{k} .
$$

Proof. By using hypothesis (D), $\bar{Q}_{I I}\left(x, U^{I}, \varepsilon U^{I I}\right)$ is uniformly bounded in $L^{\infty}$. Then $\varepsilon^{-1} Q_{I I}\left(x, U^{I}, \varepsilon U^{I I}\right)=\bar{Q}_{I I}\left(x, U^{I}, \varepsilon U^{I I}\right) U^{I I}$ is uniformly bounded in $L^{2}$; therefore, $\varepsilon^{-1} Q_{I I}\left(x, U^{I}, \varepsilon U^{I I}\right)$ is relatively compact in $H_{l o c}^{-1}$. In a similar way, thanks to the conditions on the function $D\left(U^{I}, \varepsilon U^{I I}\right)$, we get $\varepsilon^{-1} D^{I}\left(U^{I}, \varepsilon U^{I I}\right)$ and $D^{I I}\left(U^{I}, \varepsilon U^{I I}\right)$ belong to the relatively compact subset of $H_{l o c}^{-1}$. Now by using the identities

$$
\begin{aligned}
& U_{t}^{I}+M^{12}(x, D) U^{I I}=\frac{1}{\varepsilon} D^{I}\left(U^{I}, \varepsilon U^{I I}\right), \\
& M^{21}(x, D) U^{I}=\frac{1}{\varepsilon} Q\left(x, U^{I}, \varepsilon U^{I I}\right)+D^{I I}\left(U^{I}, \varepsilon U^{I I}\right)-\varepsilon^{2} U_{t}^{I I}-\varepsilon M^{22}(x, D) U^{I I},
\end{aligned}
$$

and by taking into account the previous a priori estimates of Theorem 4.2 we conclude that the family

$$
\left(\begin{array}{c}
U_{t}^{I}+M^{12}(x, D) U^{I I} \\
M^{21}(x, D) U^{I}
\end{array}\right) \quad \text { is relatively compact in }\left(H_{l o c}^{-1}\right)^{2} .
$$

Since the symbols $m^{i j}(x, \xi)$ are polihomogeneous in the sense of Section 2, we recall that the operator $M^{i j}(x, D)$ can be decomposed in the following way:

$$
M^{i j}(x, D)=M_{1}^{i j}(x, D)+M_{r}^{i j}(x, D),
$$

where $M_{1}^{i j}(x, D) \in O P S^{1}$, while $M_{r}^{i j}(x, D) \in O P S^{0}$. By applying Theorem 2.1 and by the decomposition (4.12) we get

$$
\left(\begin{array}{c}
U_{t}^{I}+M_{1}^{12}(x, D) U^{I I} \\
M_{1}^{21}(x, D) U^{I}
\end{array}\right) \quad \text { is relatively compact in }\left(H_{l o c}^{-1}\right)^{2} .
$$

In order to fit into the framework of Theorem 2.4 we write

$$
P\left[\begin{array}{c}
U^{I} \\
U^{I I}
\end{array}\right]=\left[\begin{array}{cc}
I_{k \times k} & 0 \\
0 & 0
\end{array}\right] \partial_{t}\left[\begin{array}{c}
U^{I} \\
U^{I I}
\end{array}\right]+\left[\begin{array}{cc}
0 & M_{1}^{12}(x, D) \\
M_{1}^{21}(x, D) & 0
\end{array}\right]\left[\begin{array}{c}
U^{I} \\
U^{I I}
\end{array}\right] .
$$


Then the principal symbol of $P$ is given by

$$
p(x, \eta)=\left[\begin{array}{cc}
I_{k \times k} & 0 \\
0 & 0
\end{array}\right] \xi_{0}+\left[\begin{array}{cc}
0 & m_{1}^{12}(x, \xi) \\
m_{1}^{21}(x, \xi) & 0
\end{array}\right],
$$

for any $\eta=\left(\xi, \xi_{0}\right) \in \mathbb{R}^{d+1},|\eta|=1$. We notice that

$$
p(x, \eta)\left[\begin{array}{l}
\lambda \\
\mu
\end{array}\right]=0 \Longleftrightarrow\left\{\begin{array}{l}
\xi_{0} \lambda+m_{1}^{12}(x, \xi) \mu=0, \\
m_{1}^{21}(x, \xi) \lambda=0
\end{array}\right.
$$

for all $\lambda \in \mathbb{R}^{k}, \mu \in \mathbb{R}^{N-k}$.

Now if $\xi=0$, then $\xi_{0} \neq 0$ and $m_{1}^{21}(x, 0)=0$ and so $\lambda=0$; otherwise, if $\xi \neq 0$ by using the hypothesis $(\mathrm{K})$, we get $\lambda=0$. Therefore, we get

$$
\left\{(x, \eta, \lambda, \mu) \text { such that } p(x, \eta)\left[\begin{array}{l}
\lambda \\
\mu
\end{array}\right]=0\right\} \subset\{(x, \eta, \lambda, \mu) \mid \lambda=0\} .
$$

Now we take

$$
\chi(x)=\left[\begin{array}{cc}
I_{k \times k} & 0 \\
0 & 0
\end{array}\right]
$$

and for all $\eta \neq 0, \eta=\left(\xi, \xi_{0}\right)$, we have

$$
p(x, \eta)\left[\begin{array}{l}
\lambda \\
\mu
\end{array}\right]=0 \quad \text { implies } \quad\left\langle\chi(x)\left[\begin{array}{l}
\lambda \\
\mu
\end{array}\right],\left[\begin{array}{l}
\lambda \\
\mu
\end{array}\right]\right\rangle=0,
$$

for all $\lambda \in \mathbb{R}^{k}, \mu \in \mathbb{R}^{N-k}$.

Now we can apply Theorem 2.4 of Gérard and we conclude that for any $\varphi \in$ $\mathcal{D}\left(\mathbb{R}^{d} \times \mathbb{R}_{+}\right)$,

$$
\int_{0}^{+\infty} \int_{\mathbb{R}^{d}}\left|U^{I}(x, t)\right|^{2} \varphi(x, t) d x d t \longrightarrow \int_{0}^{+\infty} \int_{\mathbb{R}^{d}}\left|U^{I 0}(x, t)\right|^{2} \varphi(x, t) d x d t,
$$

where $U^{I 0}$ denotes, in view of Theorem 4.2 the weak limit of $U^{I}$ in $L^{2}\left(\mathbb{R}^{d} \times \mathbb{R}_{+}\right)$. In this way we obtain

$$
U^{I} \longrightarrow U^{I 0} \quad \text { strongly in }\left[L_{l o c}^{2}\left(\mathbb{R}^{d} \times \mathbb{R}_{+}\right)\right]^{k} .
$$

Corollary 4.8. Assume that the hypotheses of Theorems 4.6. 4.7 hold. Then $\left(U^{I 0}, U^{I I 0}\right)$ verifies the following system, in the sense of distributions:

$$
\left\{\begin{array}{l}
U_{t}^{I 0}+M^{12}(x, D) U^{I I 0}=D_{I I}^{I}\left(U^{I 0}, 0\right) U^{I I 0} \\
M^{21}(x, D) U^{I 0}=Q_{I I}\left(x, U^{I 0}, 0\right) U^{I I 0}+D^{I I}\left(U^{I 0}, 0\right) .
\end{array}\right.
$$

Proof. By taking into account the regularity hypothesis on $Q$, the strong convergence of $\left\{U^{I}, \varepsilon U^{I I}\right\}$ in $L_{l o c}^{2}$ and the weak convergence of $U^{I I}$, we get

$$
\varepsilon^{-1} Q\left(x, U^{I}, \varepsilon U^{I I}\right) \rightarrow Q_{I I}\left(x, U^{I 0}, 0\right) U^{I I 0} \quad \text { weakly in } L_{l o c}^{2}\left(\mathbb{R}^{d} \times \mathbb{R}_{+}\right) .
$$

In a similar way we have

$$
\varepsilon^{-1} D^{I}\left(U^{I}, \varepsilon U^{I I}\right) \rightarrow D_{I I}^{I}\left(U^{I 0}, 0\right) U^{I I 0} \quad \text { weakly in } L_{\text {loc }}^{2}\left(\mathbb{R}^{d} \times \mathbb{R}_{+}\right) .
$$

Therefore, we can pass into the limit the other terms and we obtain the relations (4.14).

Remark 4.9. We can weakly relax the assumption concerning the polihomogeneity of our symbols by directly assuming the decomposition (4.12). 


\section{The Constant COefFicients CASE}

In this section we want to show how, in the case of constant coefficient differential semilinear systems, our theory can be easily simplified. First, we remark that in this case we don't need to use pseudodifferential theory; because of the constant coefficient we can handle them with classical methods. We point out that also in this case we will assume the existence of symmetrizers $R(D)$ with block structure. Since the coefficients are constant the principal symbol of $R(D)$ depends only on the variable $\xi$; more exactly it is a homogeneous radial function of degree zero of $\xi, r(\xi)=r\left(\frac{\xi}{|\xi|}\right)$. Then the symmetrizers reduce to Fourier multipliers.

5.1. A priori estimate. Here we consider the system

$$
\left\{\begin{array}{c}
U_{t}^{I}+\sum_{j=1}^{d} M_{j}^{12} \partial_{j} U^{I I}=\frac{1}{\varepsilon} D^{I}\left(U^{I}, \varepsilon U^{I I}\right), \\
\varepsilon^{2} U_{t}^{I I}+\sum_{j=1}^{d} M_{j}^{21} \partial_{j} U^{I}+\varepsilon \sum_{j=1}^{d} M_{j}^{22} \partial_{j} U^{I I} \\
=\frac{1}{\varepsilon} Q\left(x, U^{I}, \varepsilon U^{I I}\right)+D^{I I}\left(U^{I}, \varepsilon U^{I I}\right)
\end{array}\right.
$$

with the hypotheses of Section 3.2, Section 3.3, Section 3.4 and Section 4.1, specialized to our simpler framework. Namely, we assume:

(A.4bis) There exists a symmetrizer for the system (15.1), $R(D) \in O P S_{1,0}^{0}$ and its symbol $r(\xi)$ is a positive definite matrix for $|\xi|>0$.

(S.2bis) The symbol of $R(D)$ has the form

$$
r(\xi)=\left(\begin{array}{cc}
r_{11}(\xi) & 0 \\
0 & r_{22}(\xi)
\end{array}\right),
$$

where $r_{11}(\xi) \in \mathcal{M}_{k \times k}, r_{22}(\xi) \in \mathcal{M}_{(N-k) \times(N-k)}$ are symmetric positive definite matrices.

(Kbis) $\operatorname{det}\left[\left(M_{j}^{21}\right)^{T} M_{j}^{21}\right] \neq 0$ for any $j=1, \ldots, d$.

(B.2bis) $D=\left(D^{I}\left(Z^{I}, Z^{I I}\right), D^{I I}\left(Z^{I}, Z^{I I}\right)\right) \in C^{1}\left(\mathbb{R}^{N} ; \mathbb{R}^{k} \times \mathbb{R}^{N-k}\right), D^{I}\left(Z^{I}, 0\right)=$ $0, D^{I I}(0,0)=0$, and $D_{I I}^{I}$ is bounded in $\left(Z^{I}, Z^{I I}\right), D^{I I}$ is a lipschitz function in $\left(Z^{I}, Z^{I I}\right)$, with lipschitz norm $\alpha$.

(Dbis) $Q\left(x, Z^{I}, Z^{I I}\right) \in C^{1}\left(\mathbb{R}^{N+d} ; \mathbb{R}^{N-k}\right), Q\left(x, Z^{I}, 0\right)=0$ for any $\left(x, Z^{I}\right) \in \mathbb{R}^{d} \times$ $\mathbb{R}^{k}$ and $Q_{I I}\left(x, Z^{I}, Z^{I I}\right)$ is bounded in $\left(x, Z^{I}, Z^{I I}\right)$; moreover, there exists $\lambda>0$ such that $r_{22} Q_{I I}\left(x, Z^{I}, Z^{I I}\right)+\left(Q_{I I}\left(x, Z^{I}, Z^{I I}\right)\right)^{T} r_{22} \leq-\lambda r_{22}$.

Remark 5.1. (1) Obviously, the hypotheses (A.4bis), (S.2bis) are satisfied in many cases; see [2].

(2) Obviously, the hypothesis (Dbis) can be replaced by the hypothesis (D) of the variable coefficients case.

We also have in this case the following theorem.

Theorem 5.2. Let us consider the solution $\left\{U^{I}\right\},\left\{U^{I I}\right\}$ of the Cauchy problem for the system (15.1). Assume $U^{I}(x, 0) \in\left[L^{2}\left(\mathbb{R}^{d}\right)\right]^{k}, U^{I I}(x, 0) \in\left[L^{2}\left(\mathbb{R}^{d}\right)\right]^{N-k}$ and that hypotheses (A.4bis), (S.2bis), (B.2bis), (Dbis) hold. Then there exists $\varepsilon_{0}>0$ such that, for $\varepsilon \in\left(0, \varepsilon_{0}\right)$, one has

(i) for any $T>0$, there exists $M(T)>0$, independent of $\varepsilon$, such that $\left\|U^{I I}\right\|_{L^{2}\left(\mathbb{R}^{d} \times[0, T]\right)} \leq M(T)$ and $\sup _{[0, T]}\left\|\varepsilon U^{I I}(\cdot, t)\right\| \leq M(T)$, 
(ii) $\left\{\varepsilon^{2} U_{t}^{I I}\right\}$ is relatively compact in $H_{\text {loc }}^{-1}\left(\mathbb{R}^{d} \times \mathbb{R}_{+}\right)$,

(iii) $\left\{U^{I}\right\}$ is uniformly bounded, with respect to $\varepsilon$, in $L^{\infty}\left(\mathbb{R}_{+}, L^{2}\left(\mathbb{R}^{d}\right)\right)$; namely, for any $T>0$, there exists $M(T)>0$, independent of $\varepsilon$, such that $\sup _{[0, T]}\left\|U^{I}(\cdot, t)\right\| \leq M(T)$.

Proof. We apply the Fourier transform to the system (5.1) and we multiply in $L^{2}\left(\mathbb{R}^{d}\right)$ by $R(\xi) \widehat{U}$ obtaining the following inequality:

$$
\begin{aligned}
& \frac{d}{d t}\left\{\frac{\varepsilon^{2}}{2}\left|r_{22}^{1 / 2}(\xi) \widehat{U^{I I}}\right|^{2}+\left|r_{11}^{1 / 2}(\xi) \widehat{U^{I}}\right|^{2}\right\}=\left(r_{11}(\xi) \widehat{U^{I}}, \varepsilon^{-1} \widehat{D^{I}}\left(U^{I}, \varepsilon U^{I I}\right)_{2}\right. \\
& +\left(r_{22}(\xi) \widehat{U^{I I}}, \varepsilon^{-1} \widehat{Q}\left(x, U^{I}, \varepsilon U^{I I}\right)\right)_{2}+\left(r_{22}(\xi) \widehat{U^{I I}}, \widehat{D^{I I}}\left(U^{I}, \varepsilon U^{I I}\right)\right)_{2} .
\end{aligned}
$$

Defining the energy as in (4.3), taking into account the hypotheses, and using the properties of the symmetrizer and Plancharel theorem yields the standard energy inequality (4.4). The remaining part of the proof follows exactly the same arguments used in the previous section, so it is omitted.

5.2. Basic ideas on strong convergence. The analysis of strong convergence in this case reduces to analyzing the convergence of quadratic forms with constant coefficients via the classical compensated compactness result of Tartar (see [52, [53], [45]; see also [4]).

Theorem 5.3 (Tartar's compensated compactness). Let us consider

(1) a bounded open set $\Omega \subset \mathbb{R}^{n}$;

(2) a sequence $\left\{l^{\nu}\right\}_{\nu=1}^{\infty}, l^{\nu}: \Omega \subset \mathbb{R}^{n} \longrightarrow \mathbb{R}^{m}$;

(3) a symmetric matrix $\Theta: \mathbb{R}^{m} \longrightarrow \mathbb{R}^{m}$;

(4) constants $a_{j k}^{i} \in \mathbb{R}, i=1, \ldots, q, j=1, \ldots, m, k=1, \ldots, n$.

Let us define

$$
\begin{aligned}
f(\alpha) & =\langle\Theta \alpha, \alpha\rangle, \quad \text { for all } \alpha \in \mathbb{R}^{m} ; \\
\Lambda & =\left\{\lambda \in \mathbb{R}^{m}: \exists \eta \in \mathbb{R}^{n} \backslash\{0\}, \sum_{j, k} a_{j k}^{i} \lambda_{j} \eta_{k}=0, i=1, \ldots, q\right\} .
\end{aligned}
$$

Assume that

(a) there exists $\widetilde{l} \in L_{m}^{2}(\Omega)$ such that $l^{\nu} \rightarrow \widetilde{l}$ in $L_{m}^{2}(\Omega)$ as $\nu \uparrow \infty$;

(b) $\mathcal{A}^{i} l^{\nu}=\sum_{j, k} a_{j k}^{i} \frac{\partial l_{j}^{\nu}}{\partial x_{k}}, i=1, \ldots, q$, are relatively compact in $H_{l o c}^{-1}(\Omega)$;

(c) $f_{\mid \Lambda} \equiv 0$;

(d) there exists $\widetilde{f} \in \mathbb{R}$ such that $f(l) \rightarrow \widetilde{f}$ in the sense of measures $\mathcal{M}(\Omega)$.

Then we have $\tilde{f}=f(\widetilde{l})$.

Now we can state our convergence result

Theorem 5.4. Let $\left\{U^{I}, U^{I I}\right\}$ be the solution of the Cauchy problem for system (5.1). Let us assume the hypotheses (A.4bis), (S.2bis), (B.2bis), (Kbis), (Dbis) hold. Then there exist $U^{I 0} \in\left[L^{2}\left(\mathbb{R}^{d} \times[0, T]\right)\right]^{k}$ and $U^{I I 0} \in\left[L^{2}\left(\mathbb{R}^{d} \times[0, T]\right)\right]^{N-k}$ such that, as $\varepsilon \downarrow 0$ (extracting eventually subsequences),

$$
\begin{array}{ll}
U^{I I} \longrightarrow U^{I I 0} & \text { weakly in }\left[L^{2}\left(\mathbb{R}^{d} \times[0, T]\right)\right]^{k}, \\
\varepsilon U^{I I} \longrightarrow 0 & \text { strongly in }\left[L_{l o c}^{2}\left(\mathbb{R}^{d} \times \mathbb{R}_{+}\right)\right]^{N-k}, \\
U^{I} \longrightarrow U^{I 0} & \text { strongly in }\left[L_{\text {loc }}^{2}\left(\mathbb{R}^{d} \times \mathbb{R}_{+}\right)\right]^{N-k},
\end{array}
$$


and the limit profile $\left(U^{I 0}, U^{I I 0}\right)$ verifies the system

$$
\left\{\begin{array}{l}
U_{t}^{I 0}+\sum_{j=1}^{d} M_{j}^{12} \partial_{j} U^{I I 0}=D_{I I}^{I}\left(U^{I 0}, 0\right) U^{I I 0} \\
\sum_{j=1}^{d} M_{j}^{21} \partial_{j} U^{I 0}=Q_{I I}\left(x, U^{I 0}, 0\right) U^{I I 0}+D^{I I}\left(U^{I 0}, 0\right),
\end{array}\right.
$$

in the sense of distribution.

Proof. (5.2), (5.3) follow from (i) of Theorem 5.2 and the energy estimate implies that

$$
\left(\begin{array}{c}
U_{t}^{I}+\sum_{j=1}^{d} M_{j}^{12} \partial_{j} U^{I I} \\
\sum_{j=1}^{d} M_{j}^{21} \partial_{j} U^{I}
\end{array}\right)
$$

is relatively compact in $\left(H_{l o c}^{-1}\right)^{2}$.

In order to fit into the framework of Theorem 5.3 we set $l^{\varepsilon}=\left(U^{I}, U^{I I}\right)$. Then the characteristic manifold $\Lambda$ is given by

$$
\Lambda=\left\{(\lambda, \mu) \in \mathbb{R}^{k} \times \mathbb{R}^{N-k} \mid \exists \eta \in \mathbb{R}^{d+1} \backslash\{0\}, B(\eta, \lambda)=0\right\},
$$

where

$$
B(\eta, \lambda)=\left(\begin{array}{c}
\xi_{0} \lambda+\left(\sum_{j=1}^{d} M_{j}^{22} \xi_{j}\right) \mu \\
\left(\sum_{j=1}^{d} M_{j}^{21}(x) \xi_{j}\right) \lambda
\end{array}\right) .
$$

By using the hypothesis (Kbis) and by defining $\Theta=\frac{1}{2}\left(\begin{array}{r}I_{k \times k} 0 \\ 0 \\ 0\end{array}\right)$, we have $f(\lambda)=$ $\lambda^{T} \Theta \lambda$ and, of course, $f_{\mid \Lambda} \equiv 0$. We apply Theorem 5.3 to show

$$
\left(U^{I}\right)^{2} \rightarrow\left(U^{I 0}\right)^{2} \quad \text { in the sense of measure, }
$$

and finally,

$$
U^{I} \longrightarrow U^{I 0} \quad \text { strongly in }\left[L_{\text {loc }}^{2}\left(\mathbb{R}^{d} \times \mathbb{R}_{+}\right)\right]^{k} .
$$

So we can pass to the limit into the nonlinear terms of the system (5.1).

Remark 5.5. An equivalent form of system (5.5) is given by (setting $U^{I 0}=U$ )

$$
\begin{aligned}
U_{t} & +\sum_{j=1}^{d} M_{j}^{12} \partial_{j}\left(Q_{I I}^{-1}(x, U, 0) \sum_{k=1}^{d} M_{k}^{21} \partial_{k} U\right) \\
& =\sum_{j=1}^{d} M_{j}^{12} \partial_{j}\left(Q_{I I}^{-1}(x, U, 0) D^{I I}(U, 0)\right) \\
& +D_{I I}^{I}\left(U^{I}, 0\right) Q_{I I}^{-1}(x, U, 0)\left[\sum_{k=1}^{d} M_{k}^{21} \partial_{k} U-D^{I I}(U, 0)\right] .
\end{aligned}
$$

The proof of the parabolicity of (5.6) follows the same arguments used in the variable coefficients case. 


\section{Approximation of PRESCRIBED PARABOLIC SYSTEMS}

In this last section we want to apply the theory of the previous sections to approximate a generic given parabolic system, provided the Petrowski condition holds. In fact, here we reconstruct a parabolic system by means of a suitable larger semilinear hyperbolic system that relaxes on it. Two very large classes of parabolic systems will be taken into consideration. The first are quasilinear parabolic systems in divergence form and the second are the so-called "Reaction-Diffusion" systems.

6.1. Quasilinear case. We consider now the following quasilinear system in divergence form:

$$
U_{t}+\sum_{i=1}^{d} \partial_{i}\left(F_{i}(U)-\sum_{j=1}^{d} B_{i j}(U) \partial_{j} U\right)=G(U)
$$

where $x \in \mathbb{R}^{d}, t \in \mathbb{R}_{+}, U=U(x, t) \in \mathbb{R}^{k}, N=(d+1) k$ (then $k \leq \frac{N}{2}$ ). Let us denote $\mathbb{F}(U) \in \mathcal{M}_{k \times d},(\mathbb{F}(U))_{i}=F_{i}(U)$ for any $i=1, \ldots, d$ and $\mathbb{B}(U) \in \mathcal{M}_{k d \times k d}$, $(\mathbb{B}(U))_{i j}=B_{i j}(U)$ for any $i, j=1, \ldots, d$. Then we assume:

(C.1) $B_{i j}(\cdot) \in C^{1}\left(\mathbb{R}^{k} ; \mathcal{M}_{k \times k}\right)$, for any $i, j=1, \ldots, d$ and $\sum_{i, j}\left(B_{i j}+\left(B_{i j}\right)\right)^{T}(U) \lambda_{i} \lambda_{j}$ $\geq c_{0}|\lambda|^{2} I$ for any $\lambda \in \mathbb{R}^{d}$ (strong parabolicity); moreover, $\mathbb{B}^{-1}(U)$ is bounded on $U$.

(C.2) $F_{i}(\cdot) \in C^{1}\left(\mathbb{R}^{k} ; \mathbb{R}^{k}\right)$, for any $i=1, \ldots, d, \mathbb{F}(0)=0$ and $\mathbb{B}^{-1}(U) \mathbb{F}(U)$ is lipschitz on $U$.

(C.3) $G(\cdot) \in \operatorname{Lip}\left(\mathbb{R}^{k} ; \mathbb{R}^{k}\right)$.

We have the following theorem.

Theorem 6.1. Let us consider the system (6.1), and let us suppose hypotheses (C.1), (C.2), (C.3) hold. Then the solutions of the system

$$
\left\{\begin{array}{l}
Z_{t}^{I}+\frac{1}{\varepsilon} \operatorname{div} \mathbb{Z}^{I I}=G\left(Z^{I}\right), \\
\mathbb{Z}_{t}^{I I}+\frac{1}{\varepsilon} D Z^{I}=-\frac{1}{\varepsilon^{2}} \mathbb{B}^{-1}\left(Z^{I}\right) \mathbb{Z}^{I I}+\frac{1}{\varepsilon} \mathbb{B}^{-1}\left(Z^{I}\right) \mathbb{F}\left(Z^{I}\right),
\end{array}\right.
$$

where $(x, t) \in \mathbb{R}^{d} \times \mathbb{R}_{+}, Z^{I}=Z^{I}(x, t) \in \mathbb{R}^{k}, \mathbb{Z}^{I I}=\mathbb{Z}^{I I}(x, t) \in \mathcal{M}_{k \times d}$, approximate the system (6.1) in the sense of Theorem 4.6 and Theorem 4.7.

Proof. It can be easily shown that (6.2) is a hyperbolic symmetric system. By rescaling the variables, as in (3.12), the system (6.2) transforms into

$$
\left\{\begin{array}{l}
U_{t}^{I}+\operatorname{div} \mathbb{U}^{I I}=G\left(U^{I}\right) \\
\varepsilon^{2} \mathbb{U}_{t}^{I I}+D U^{I}=-\mathbb{B}^{-1}\left(U^{I}\right) \mathbb{U}^{I I}+\mathbb{B}^{-1}\left(U^{I}\right) \mathbb{F}\left(U^{I}\right)
\end{array}\right.
$$

The conditions (B.1), (K) of Section 4 are satisfied by the system (6.3). Now by setting $D^{I I}\left(Z^{I}, \mathbb{Z}^{I I}\right)=\mathbb{B}^{-1}\left(Z^{I}\right) \mathbb{F}\left(Z^{I}\right)$ we can easily verify (B.2), and by denot$\operatorname{ing} Q\left(x, Z^{I}, \mathbb{Z}^{I I}\right)=-\mathbb{B}^{-1}\left(Z^{I}\right) \mathbb{Z}^{I I}$ we obviously get condition (D). We can apply Theorem 4.7 and we obtain that the solutions to (6.3) satisfy, as $\varepsilon \downarrow 0$ (setting $\left.U^{I 0}=U\right)$,

$$
U_{t}+\operatorname{div}(\mathbb{F}(U)-\mathbb{B}(U) D U)=G(U) .
$$


6.2. Chemotaxis. We consider the following quasilinear system modeling chemotaxis [23]:

$$
\left\{\begin{array}{l}
u_{t}=\nabla \cdot(\nabla u-u \nabla v) \\
v_{t}=\Delta v+u
\end{array}\right.
$$

where $x \in \mathbb{R}^{d}, t \in \mathbb{R}_{+}, u=u(x, t) \in \mathbb{R}, v=v(x, t) \in \mathbb{R}$. By following the notation of Section 2, we have that the principal symbol of (6.4) is given by

$$
L(x, t, u, \xi)=-\left(\begin{array}{cc}
1 & -u \\
0 & 1
\end{array}\right)|\xi|^{2} .
$$

It can be easily verified that the system (6.4) is Petrowski parabolic in the sense of Section 2. With the theory developed in the previous sections we can try to approximate it. Let us denote $Z^{I}=\left(\begin{array}{l}u \\ v\end{array}\right) \in \mathbb{R}^{2}, \mathbb{B}\left(Z^{I}\right)=\left(\begin{array}{cc}I & -u I \\ 0 & I\end{array}\right) \in \mathcal{M}_{2 d \times 2 d}$, $I=I_{d \times d}, \mathbb{Z}^{I I}=Z^{I I}(x, t) \in \mathcal{M}_{2 \times d}, G\left(Z^{I}\right)=\left(\begin{array}{l}0 \\ u\end{array}\right) \in \mathbb{R}^{2}$. Then the system

$$
\left\{\begin{array}{l}
Z_{t}^{I}+\frac{1}{\varepsilon} \operatorname{div} \mathbb{Z}^{I I}=G\left(Z^{I}\right), \\
\mathbb{Z}_{t}^{I I}+\frac{1}{\varepsilon} D Z^{I}=-\frac{1}{\varepsilon^{2}} \mathbb{B}^{-1}\left(Z^{I}\right) \mathbb{Z}^{I I}
\end{array}\right.
$$

is the approximating system of (6.4). In order to apply our theory we need to assume the following condition on the solution $\left(Z^{I}, \mathbb{Z}^{I I}\right)$ of $(6.5)$ and (6.4):

(B) The solution $Z^{I}$ of the system (6.5) is bounded.

This allows us to circumvent condition (d1) of Section 3 which is not satisfied by $\mathbb{B}^{-1}\left(Z^{I}\right)$, which has linear growth in $Z^{I}$. We have the following theorem.

Theorem 6.2. Let us consider the system (6.4), and let us suppose hypothesis (B) holds. Then this solution of system (6.5) approximates the system (6.4) in the sense of Theorem 4.6 and Theorem 4.7.

The proof of the theorem follows the same lines as the proof of Theorem 6.2 and it will be omitted.

Remark 6.3. The Brusselator model [31, [47] can be investigated in a very similar way.

6.3. Reaction-diffusion type systems. Let us consider the reaction-diffusion type system

$$
U_{t}=\sum_{j, l=1}^{d} A_{j, l}(x) \partial_{j} \partial_{l} U+f(U),
$$

where $x \in \mathbb{R}^{d}, t \in \mathbb{R}_{+}, U=U(x, t) \in \mathbb{R}^{k}$. We make the following hypotheses:

(D.1) $A_{j, l}(\cdot) \in C^{\infty}\left(\mathbb{R}^{d} ; \mathcal{M}_{k \times k}\right)$, for any $j, l=1, \ldots, d$, and $\sum_{j, l=1}^{d} A_{j, l}(x) \lambda_{j} \lambda_{l} \geq$ $c_{0}|\lambda|^{2} I$, for any $\lambda \in \mathbb{R}^{d}$ (strong parabolicity).

(D.2) $f(\cdot) \in \operatorname{Lip}\left(\mathbb{R}^{k} ; \mathbb{R}^{k}\right)$.

In order to approximate system (6.6), we define the linear operator $B_{j}(x): \mathcal{M}_{k \times d}$ $\longrightarrow \mathcal{M}_{k \times d}$ :

$$
B_{j}(x)(W)=\sum_{l=1}^{d} A_{j, l}(x) W_{l} \quad \text { for any } j=1, \ldots, d \text {. }
$$


Now system (6.6) can be written in the equivalent form

$$
U_{t}=\sum_{j=1}^{d} B_{j}\left(\partial_{j} D U\right)+f(U) .
$$

We have the following theorem.

Theorem 6.4. Let us consider the system (6.6), and let us suppose hypotheses (D.1) and (D.2) hold. Then

$$
\left\{\begin{array}{l}
Z_{t}^{I}+\frac{1}{\varepsilon} \sum_{j=1}^{d} B_{j} \partial_{j} \mathbb{Z}^{I I}=f\left(Z^{I}\right) \\
\mathbb{Z}_{t}^{I I}+\frac{1}{\varepsilon} \sum_{j=1}^{d} B_{j}^{T} \partial_{j} Z^{I}=-\frac{1}{\varepsilon^{2}} \mathbb{A}(x) \mathbb{Z}^{I I}
\end{array}\right.
$$

where $(x, t) \in \mathbb{R}^{d} \times \mathbb{R}_{+}, Z^{I}=Z^{I}(x, t) \in \mathbb{R}^{k}, \mathbb{Z}^{I I}=\mathbb{Z}^{I I}(x, t) \in \mathcal{M}_{k \times d}, \mathbb{A}(x) \in$ $\mathcal{M}_{k d \times k d},(\mathbb{A}(x))_{j, k}=A_{j, k}(x)$ approximate the system (6.6) in the sense of Theorem 4.6 and Theorem 4.7 .

Proof. The system (6.7) is symmetric and hyperbolic. By rescaling $\mathbb{Z}^{I I}=\varepsilon \mathbb{U}^{I I}$ as in (3.12) it transforms into

$$
\left\{\begin{array}{l}
U_{t}^{I}+\sum_{j=1}^{d} B_{j} \partial_{j} \mathbb{U}^{I I}=f\left(U^{I}\right) \\
\varepsilon^{2} \mathbb{U}_{t}^{I I}+\sum_{j=1}^{d} B_{j}^{T} \partial_{j} U^{I}=-\mathbb{A}(x) \mathbb{U}^{I I}
\end{array}\right.
$$

Hence, by setting $Q\left(x, Z^{I}, \mathbb{Z}^{I I}\right)=-\mathbb{A}(x) \mathbb{Z}^{I I}$, the dissipativity conditions (D) together with the hypotheses (B.1) and (K) of Section 4 are immediately satisfied. Now, we can apply Theorems 4.6 and 4.7. Then since $\sum_{j=1}^{d} B_{j}^{T} \partial_{j} U^{I}=\mathbb{A}(x) D U^{I}$, as $\varepsilon \downarrow 0$, the solution of (6.8) satisfies the system (6.6).

Remark 6.5. Theorem 6.4 can be applied, with slight modifications, to the more general case of Petrowski parabolic systems in the sense recalled in Section 2. In fact, let us consider the system

$$
U_{t}=\sum_{j, k=1}^{d} C_{j, k}(x) \partial_{j} \partial_{k} U+G(U),
$$

where $x \in \mathbb{R}^{d}, t \in \mathbb{R}_{+}, U=U(x, t) \in \mathbb{R}^{k}$ and the matrices $C_{j, k}$ satisfy the Petrowski parabolicity condition given in Section 2. Let us consider the matrix $P_{0}$ from the previous definition, and denote

$$
W=P_{0} U
$$

Then, the system (6.9) transforms into a strongly parabolic system; so we can apply Theorem 6.4.

To approximate system (6.6) we can also follow a different approach by using pseudodifferential theory. In fact, let us denote by

$$
A(x, \xi)=-\sum_{j, k} A_{j, k}(x) \xi_{j} \xi_{k}
$$


$\xi \in \mathbb{R}^{d}$, the principal symbol of ([6.6), and set $A(x, D)=O P A(x, \xi)$. Now we set

$$
B(x, D)=O P\left[(-A(x, \xi))^{1 / 2}\right] .
$$

We have the following theorem.

Theorem 6.6. Let us consider system (6.6), and suppose that hypotheses (D.1) and (D.2) hold. Then the solutions of the system

$$
\left\{\begin{array}{l}
Z_{t}^{I}+\frac{1}{\varepsilon} B(x, D) Z^{I I}=f\left(Z^{I}\right), \\
Z_{t}^{I I}-\frac{1}{\varepsilon} B(x, D) Z^{I}=-\frac{1}{\varepsilon^{2}} Z^{I I},
\end{array}\right.
$$

where $(x, t) \in \mathbb{R}^{d} \times \mathbb{R}_{+}, Z^{I}=Z^{I}(x, t) \in \mathbb{R}^{k}, Z^{I I}=Z^{I I}(x, t) \in \mathbb{R}^{k}, N=2 k$, approximate system (6.6) in the sense of Theorem 4.6 and Theorem 4.7.

Proof. By rescaling the variables as in (3.12), the system (6.6) transforms into

$$
\left\{\begin{array}{l}
U_{t}^{I}+B(x, D) U^{I I}=f\left(U^{I}\right), \\
\varepsilon^{2} U_{t}^{I I}-B(x, D) U^{I}=-U^{I I} .
\end{array}\right.
$$

By using (D.1) we have that (6.13) is a hyperbolic system. Let us denote $M^{12}(x, D)=B(x, D), M^{21}(x, D)=-B(x, D)$; by using hypothesis (D.1), conditions (B.1) and (K) are satisfied. It can also be easily verified that the symmetrizer of (6.12) is given by the matrix $I_{N \times N}$. Finally, we have to verify the dissipativity condition (D). Setting $Q\left(x, Z^{I}, Z^{I I}\right)=-Z^{I I}$ we get that hypothesis (D) is satisfied. Now we can apply Theorem 4.6 and Theorem 4.7 and we obtain that the solution of (6.13) satisfies, as $\varepsilon \downarrow 0$ (by setting $U^{I 0}=U$ ),

$$
U_{t}-A(x, D) U=0 .
$$

\section{Appendix A. Formal asymptotics on Condition (S.1)}

In the case where (S.1) does not hold, we cannot expect a relaxation from hyperbolic to parabolic systems, but instead we will have a multiscale phenomena, involving the simultaneous action of distinct relaxation mechanisms. To clarify this fact, we follow the formal asymptotic approximations of Lattanzio and Yong [29]; namely, we consider the scaled system

$$
W_{t}+\frac{1}{\varepsilon} \sum_{j=1}^{d} A_{j}(x) W_{x_{j}}=\frac{B(W)}{\varepsilon^{2}},
$$

where $W \in \mathbb{R},(x, t) \in \mathbb{R}^{d} \times \mathbb{R}_{+}$, and $A_{j}(x)$ are smooth $N \times N$ matrices, with the following assumption:

(a) the first $k$ components of $B(W)$ are zero; that is,

$$
B(W)=\left(\begin{array}{c}
0 \\
Q(W)
\end{array}\right)
$$

with $Q(W) \in \mathbb{R}^{N-k}$. 
Corresponding to this decomposition of $Q$, we set

$$
W=\left(\begin{array}{l}
u \\
v
\end{array}\right), \quad A_{j}=\left(\begin{array}{cc}
A_{j}^{11} & A_{j}^{12} \\
A_{j}^{21} & A_{j}^{22}
\end{array}\right) .
$$

Furthermore, we assume:

(b) $Q(u, v)=0$ if and only if $v=0, Q_{v}(u, 0)$ is invertible for any $u$.

(c) $A_{j}(x) \neq 0$ for any $x \in \mathbb{R}^{d}$, for all $j=1, \ldots, d$.

We look for a solution of the form

$$
O_{\varepsilon}(x, t) \sim \sum_{k=0}^{\infty} \varepsilon^{k} O_{k}(x, t)=O_{0}(x, t)+\sum_{k=1}^{\infty} \varepsilon^{k} O_{k}(x, t) .
$$

Recalling (A.1) we introduce

$$
R(W)=W_{t}+\frac{1}{\varepsilon} \sum_{j=1}^{d} A_{j}(x) W_{x_{j}}-\frac{B(W)}{\varepsilon^{2}} .
$$

Since $O_{\varepsilon}$ is expected to solve (A.1) we want $R\left(O_{\varepsilon}\right)=0$. Thus it follows that

$$
\begin{aligned}
B\left(O_{0}\right) & =0, \\
\sum_{j=1}^{d} A_{j}(x) O_{0 x_{j}} & =B_{W}\left(O_{0}\right) O_{1}, \\
O_{k t}+\sum_{j=1}^{d} A_{j}(x) O_{k+1, x_{j}} & =B_{W}\left(O_{0}\right) O_{k+2} .
\end{aligned}
$$

Set $O_{k}=\left(\begin{array}{l}u_{k} \\ v_{k}\end{array}\right)$. The above equations can be rewritten as

$$
\begin{aligned}
& Q\left(u_{0}, v_{0}\right)=0 \\
& \sum_{j=1}^{d}\left(A_{j}^{11}(x) u_{0 x_{j}}+A_{j}^{12}(x) v_{0 x_{j}}\right)=0 \\
& \sum_{j=1}^{d}\left(A_{j}^{21}(x) u_{0 x_{j}}+A_{j}^{22}(x) v_{0 x_{j}}\right)=Q_{u}\left(u_{0}, v_{0}\right) u_{1}+Q_{v}\left(u_{0}, v_{0}\right) v_{1}, \\
& u_{k t}+\sum_{j=1}^{d}\left(A_{j}^{11}(x) u_{k+1, x_{j}}+A_{j}^{12}(x) v_{k+1, x_{j}}\right)=0, \\
& v_{k t}+\sum_{j=1}^{d}\left(A_{j}^{21}(x) u_{k+1, x_{j}}+A_{j}^{22}(x) v_{k+1, x_{j}}\right)=Q_{u}\left(u_{0}, v_{0}\right) u_{k+2} \\
& +Q_{v}\left(u_{0}, v_{0}\right) v_{k+2} .
\end{aligned}
$$

From (A.2) and (b) it follows that $v_{0}=0$. Then (A.3) reduces to

$$
\sum_{j=1}^{d} A_{j}^{11}(x) u_{0 x_{j}}=0
$$


so by using (c) we get $u_{0 x_{j}}=0$. By using (b) and A.4 we get $v_{1}=0$ and setting $k=0$ in A.5 implies $u_{1}=0$. Up to now we have found $u_{0}=v_{0}=u_{1}=v_{1}=0$. By assuming inductively $u_{p}=v_{p}=0$ and by using the previous relations and (b) we get $u_{p+1}=v_{p+1}=0$. Hence, the formal limit is the null solution.

\section{REFERENCES}

1. F. Bouchut, F. Guarguaglini and R. Natalini, Diffusive BGK approximation for nonlinear multidimensional parabolic equations. Indiana Univ. Math. J., 49 (2000), no. 2, 723-749. MR 2001k:35162

2. J. Chazarain, A. Piriou, Introduction to the theory of linear partial differential equations. Studies in Mathematics and its Applications, 14 (1982), North-Holland Pub. MR 83j:35001

3. G.-Q. Chen, C.D. Levermore, and T.-P. Liu, Hyperbolic conservation laws with stiff relaxation terms and entropy. Comm. Pure Appl. Math., 47 (1994), no. 6, 787-830. MR 95h:35133

4. B. Dacorogna, Weak Continuity and Weak Lower Semicontinuity of Nonlinear Functional. Springer-Verlag, 1982. MR 84f:49020

5. C. Dafermos, Hyperbolic conservation laws in continuum physics. Grundlehren der mathematischen Wissenschaften 325 (2000), Springer-Verlag. MR 2001m:35212

6. P. Degond, T. Goudon, F.Poupaud, Diffusion limit for nonhomogeneous and non-microreversible processes. Indiana Univ. Math. J. 49 (2000), no. 3, 1175-1198. MR 2002a:35012

7. R.J. DiPerna, Convergence of approximate solutions to conservation laws. Arch. Rational Mech. Anal., 82 (1983), no. 1, 27-70. MR 84k:35091

8. R.J. DiPerna. Compensated compactness and general systems of conservation laws. Trans. Amer. Math. Soc., 292 (1985), no.2, 383-420. MR 87g:35148

9. D. Donatelli, P. Marcati, Relaxation of semilinear hyperbolic systems with variable coefficients. Ricerche di Matematica, 48 (1999), suppl., 295-310. MR 2001d:35129

10. D. Donatelli, P. Marcati, $1-\mathcal{D}$ Relaxation from hyperbolic to parabolic systems with variable coefficients. Rendiconti dell' Istituto di Matematica dell'Università di Trieste, 31 (2000), suppl., 63-85. MR 2001j:35182

11. S.D. Eidel'man, Parabolic Systems. North-Holland Publishing Company (1969). MR 40:6023

12. L.C. Evans, Weak Convergence Methods for Nonlinear Partial Differential Equations. CBMS Regional Conference series in Mathematics of AMS, 74 (1990). MR 91a:35009

13. P. Gérard, Microlocal defect measures. Comm. Partial Differential Equations, 16 (1991), no. 11, 1761-1794. MR 92k:35027

14. F. Golse, L. St. Raymond, The Navier-Stokes limit of the Boltzmann equation: convergence proof. Preprint R01035, Laboratoire d'Analyse Numérique, Univ. Paris VI, 2001.

15. L. Hörmander, The analysis of linear partial differential operators. Grundleheren der matematischen Wissenschaften, vols. I-IV, Springer-Verlag, 1983-1985. MR 85g:35002b

16. S. Jin, H. L. Liu, Diffusion limit of a hyperbolic system with relaxation. Meth. and. Appl. Anal., 5, (1998), 317-334. MR 2000k:35176

17. S. Jin, L. Pareschi, G. Toscani Uniformly accurate diffusive relaxation schemes for multiscale transport equations. SIAM J. Numer. Anal. 38 (2000), no. 3, 913-936. MR 2001h:65095

18. M. Junk, W.A. Yong, Rigorous Navier-Stokes Limit of the Lattice Boltzmann Equation, Technical Report, IWR, Universität Heidelberg.

19. M.A. Katsoulakis and A.E. Tzavaras, Contractive relaxation systems and interacting particles for scalar conservation laws. C. R. Acad. Sci. Paris S. I Math., 323 (1996), no. 8, 865-870. MR 97g:35104

20. M.A Katsoulakis and A.E. Tzavaras, Contractive relaxation systems and the scalar multidimensional conservation law. Comm. Partial Differential Equations, 22 (1997), no. 1-2, 195233. MR 97m:35168

21. M.A. Katsoulakis and A.E. Tzavaras, Multiscale analysis for interacting particles: relaxation systems and scalar conservation laws. J. Statist. Phys. 96 (1999), no. 3-4, 715-763. MR 2000j:82029

22. S. Kawashima, Large-time behaviour of solutions to hyperbolic-parabolic systems of conservation laws and applications. Proc. Roy. Soc. Edinburg Sect. A, 106 (1987), no. 1-2, 169-194. MR 89d:35022

23. E. F. Keller, L. A. Segel, Initiation of slime mold aggregation viewed as instability. J. Theor. Biol. 1970, 399-415. 
24. H.-0. Kreiss, Initial-boundary Value Problems for Hyperbolic systems. Comm. on Pure and Applied Math., 23 (1970), 277-298. MR 55:10862

25. H.-0. Kreiss, J. Lorenz, Initial-boundary Value Problems and the Navier-Stokes Equations. Academic Press (1989). MR 91a:35138

26. T.G. Kurtz, Convergence of sequences of semigroups of nonlinear operators with an application to gas kinetics. Trans. Amer. Math. Soc.,186 (1973),259-272. MR 49:1256

27. C. Lattanzio, P. Marcati, The zero relaxation to the drift-diffusion system for the 3-D isentropic Euler-Poisson model for semiconductors. Discrete Contin. Dynam. Systems, 5 (1999), no. 2, 449-455. MR 99m:35251

28. C. Lattanzio, R. Natalini, Convergence of diffusive BGK approximation for parabolic systems. Proceedings of the Royal Society of Edinburg, 132, (2002), no. 2, 341-358. MR 2003e:35136

29. C. Lattanzio, W.-A. Yong, Hyperbolic-Parabolic singular limits for first order nonlinear systems. Comm. Partial Differential Equations (to appear).

30. P.D. Lax, Shock waves and entropy, Contributions to Nonlinear Functional Analysis, 603-634. Academic Press, New York 1971. MR 52:14677

31. R. Lefever, G. Nicolis, Chemical instabilities and sustained oscillations. Journal of theoretical Biology, 30, (1971), 267.

32. J.L. Lions, Perturbations singulieres dans les problèmes aux limites et en controle optimale, 323 (1973), Springer-Verlag, Berlin. MR 58:29078

33. P.L. Lions, G. Toscani, Diffusive limit for finite velocity Boltzmann kinetic models. Rev. Mat. Iberoamericana, 13 (1997), no. 3, 473-513. MR 99g:76127

34. T.-P. Liu, Hyperbolic conservation laws with relaxation. Comm. Math. Phys., 108 (1987), no. 1, 153-175. MR 88f:35092

35. A. Majda, Compressible Fluid Flow and Systems of Conservation Laws in Several Space Dimensions. Appl. Math. Sci., 53 (1984), Springer-Verlag. MR 85e:35077

36. A. Majda and S. Osher, Initial-boundary Value Problems for hyperbolic Equations with Uniformly Characteristics Boundary. Comm. on Pure and Applied Math., 28 (1975), no. 5, 607-675. MR 53:13857

37. P. Marcati, A. Milani, The one-dimensional Darcy's law as the limit of a compressible Euler flow. J. Differential Equations, 84 (1990), no. 1, 129-147. MR 91i:35156

38. P. Marcati, A. Milani, P. Secchi, Singular convergence of weak solutions for a quasilinear nonhomogeneous hyperbolic system. Manuscripta Math., 60 (1988), no. 1, 49-69. MR 89f:35127

39. P. Marcati, R. Natalini, Weak solutions to a hydrodynamic model for semiconductors and relaxation to the drift-diffusion equation. Arch. Rational Mech. Anal., 129 (1995), no. 2, 129-145. MR 96b:65088

40. P. Marcati, R. Natalini, Weak solutions to a hydrodynamic model for semiconductors: the Cauchy problem. Proc. Roy. Soc. Edinburgh Sect. A, 125 (1995), no. 1, 115-131. MR 95k:35203

41. P. Marcati, B. Rubino, Hyperbolic to parabolic relaxation theory for quasilinear first order systems. J. Differential Equations, 162 (2000), no. 2, 359-399. MR 2001d:35125

42. H.P. McKean, The central limit theorem for Carleman's equation. Israel J. Math., 21 (1975), no. 1, 54-92. MR 54:11529

43. G. Métivier, The block structure condition for symmetric hyperbolic systems. Bull. London Math. Soc., 32 (2000), no. 6, 689-702. MR 2001i:35198

44. S. Mizohata, The theory of partial differential equations, Cambridge University Press, (1973). MR 58:29033

45. F. Murat, Compacité par compensation. Ann. Scuola Norm. Sup. Pisa Cl. Sci.(4), 5 (1978), no. 3, 489-507. MR 80h:46043a

46. G. Naldi, L. Pareschi, Numerical schemes for hyperbolic systems of conservation laws with stiff diffusive relaxation. Preprint.

47. G. Nicolis, I. Prigogine, Self-organizations in non-linear equilibrium systems, New York, Wiley-Interscience, (1971) MR 58:25436

48. J. V. Ralston, Note on a paper of Kreiss. Comm. on Pure and Applied Math., 24 (1971), no. 6, 759-762. MR 58:29326

49. B. Rubino, Weak solutions to quasilinear wave equations of Klein-Gordon or sine-Gordon type and relaxation to reaction-diffusion equations. Nonlinear Differential Equations and Appl., 4 (1997), no. 4, 439-457. MR 99f:35092 
50. D. Serre, Systèmes de lois de conservation. I, II Diderot Editeur, Paris, 1996. MR 99b:35139, MR 99e:35144

51. D. Serre, Relaxation semi-linéaire et cinetique des systìes de lois de conservation, Ann. Inst. H. Poincaré Anal. Non Linéaire, 17, (2000), 169-192. MR 2001g:35159

52. L. Tartar, Compensated compactness and applications to partial differential equations, Research Notes in Math., 39 (1979), 136-210. MR 81m:35014

53. L. Tartar, The compensated compactness method applied to partial differential equations. Systems of Nonlinear Partial Differential Equations, Reidel, Dordrecht, 1983. NATO ASI. MR 85e:35079

54. L. Tartar, H-measures, a new approach for studying homogenization and concentration effects in partial differential equations. Proc. Roy. Soc. Edinburg Sect. A, 115 (1990), no. 3-4, 193230. MR 91h:35042

55. M.E. Taylor, Pseudodifferential Operators. volume 34 Princeton Mathematical Series, 34 (1981), Princeton University Press, Princeton, New Jersey. MR 82i:35172

56. M.E. Taylor, Pseudodifferential Operators and Nonlinear PDE. Progress in Mathematics, 100 (1991), Birkäuser. MR 92j:35193

57. M.E. Taylor, Partial Differential Equations I, II, III. Appl. Math. Sciences, 115-117 (1996), Springer-Verlag. MR 98b:35002b MR 98b:35003 MR 98k:35001

58. W.-A. Yong, Singular perturbations of first-order hyperbolic systems with stiff source terms. J. Differential Equations, 155 (1999), no. 1, 89-132. MR 2000c:35011

Dipartimento di Matematica Pura ed Applicata, Università degli Studi dell'Aquila, 67100 L'Aquila, ItALY

E-mail address: donatell@univaq.it

Dipartimento di Matematica Pura ed Applicata, Università degli Studi dell'Aquila, 67100 L'Aquila, ITALY

E-mail address: marcati@univaq.it 